\title{
On the genesis of the Cartan-Kähler theory
}

\author{
Alberto Cogliati*
}

\section{Contents}

1 Introduction 2

2 Some technical preliminaries $\quad 2$

$\begin{array}{lll}3 & \text { The state of the art in the early 1890's } & 6\end{array}$

4 Engel's invariants theory of Pfaffian systems $\quad 7$

4.1 Invariant correspondences . . . . . . . . . . . . 7

5 Von Weber's contributions: 1898-1900 12

5.1 Character and characteristic transformations . . . . . . . . . 13

5.2 Pfaffian systems of character one, I . . . . . . . . . . 15

5.3 Reducibility of a Pfaffian system to its normal form . . . . . . 18

6 The foundations of the exterior differential calculus 22

7 Cartan's theory of general Pfaffian systems 25

7.1 Geometrical representation . . . . . . . . . . . . . . 25

7.2 Cauchy's first theorem . . . . . . . . . . . . . . . . . . 29

7.3 Genre and characters . . . . . . . . . . . . . . . 33

7.4 Characteristic elements . . . . . . . . . . . . . . . . . . . . . . . . . . . 37

7.5 Pfaffian systems of character one, II . . . . . . . . . . . . . 39

8 Final remarks $\quad 42$

${ }^{*}$ Università degli Studi di Milano, Dipartimento di Matematica Federigo Enriques, Via Saldini 50, 20133, Milano. E-mail: alberto.cogliati@unimi.it 


\section{Introduction}

Historians and mathematicians are unanimous in considering Cartan's work on Pfaffian systems (what nowadays we would call exterior differential systems) as a landmark both for what concerns his entire mathematical production and the development itself of 20th century mathematics. The strategic role played by such systems in so many realms of mathematical research such as general theory of partial differential equations, continuous infinite Lie groups, theory of equivalence and differential geometry, to mention only a few, is unitedly acknowledged.

Cartan himself was quite definite in assessing the importance of his work on total differential equations within his whole mathematical activity. Referring to the years when his attention was concentrated for the large part on developing his ideas on Pfaffian equations, he once wrote that those were years of calm and long meditation in which all the germs of his subsequent works were contained ${ }^{1}$.

Nevertheless, it appears that scarce attention has been paid to this area of historical research thus far. Authoritative scholars ${ }^{2}$ dealt with Pfaff's problem and the foundation of exterior differential calculus in Cartan's early papers; however no specific analysis of his subsequent works laying the foundations of what nowadays is known as the Cartan-Kähler theory has been provided so far. The present article represents a first partial attempt to remedy this unsatisfactory state of affairs ${ }^{3}$.

\section{Some technical preliminaries}

This section is devoted to some general remarks on the mathematics which we are about to deal with; historical accuracy will not be main focus of attention here, we will limit ourselves to give the necessary information that will be helpful in understanding the discussion that is going to follow. The interested reader can find a detailed historical account of this material in the paper [20] by T. Hawkins.

The main topic of our discussion will be the problem of integration of differential systems of Pfaffian equations; thus, it seems appropriate to de-

\footnotetext{
${ }^{1}$ See Appendix C in [1]: “[...] Je garde le meilleur souvenir des quinze ans que j'ai passés en province, à Montpellier d'abord, à Lyon, et à Nancy ensuite. Ce furent des années de méditation dans la calme, et tout ce que j'ai fait plus tard est contenu en germe dans mes travaux mûrement médités de cette période."

${ }^{2}$ See $[20]$ and [22].

${ }^{3}$ I wish to express my gratitude to Professor P. J. Olver for precious advices and remarks on a preliminary version of this paper.
} 
scribe briefly what a Pfaffian equation is and what it means to integrate such equations. Moreover, it is useful to emphasize a crucial separation that has to be operated in the theory and that will be of primary importance for our purposes, that is the distinction between the completely integrable (unbeschränkt integrable) case and the not completely integrable one.

In modern terms, what nineteenth century mathematicians meant by a Pfaffian form in $n$ variables can be identified with the local expression of a differential 1-form defined on a $n$-dimensional manifold. However, until 1899, when Cartan gave a symbolic definition of what he named differential expression (expression différentielle), it appears that no autonomous status was attributed to it. Rather, what was considered to be meaningful was the problem of its vanishing on suitable regions of the space. This was interpreted as the manifestation of certain finite relations (to be determined) among the independent variables.

Thus, a Pfaffian equation in $n$ variables is a differential relation of the following type:

$$
\omega=A_{1}\left(x_{1}, \cdots, x_{n}\right) d x_{1}+\cdots+A_{n}\left(x_{1}, \cdots, x_{n}\right) d x_{n}=0 .
$$

To find integrals ${ }^{4}$ of (1) means to determine, functionally independent, finite relations among the variables $x_{1}, \cdots, x_{n}, f_{j}\left(x_{1}, \cdots, x_{n}\right)=0,(j=1, \cdots, m)$ such that the vanishing of (1) is a consequence of the $2 m$ relations $f_{j}=$ $0, d f_{j}=\sum_{k=1}^{n} \frac{\partial f_{j}}{\partial x_{k}} d x_{k}=0(j=1, \cdots, m)$. These integrals can be thought of geometrically as defining an integral submanifold of dimension $n-m$ given by the intersection of $m$ hypersurfaces $f_{j}=0^{5}$.

During the nineteenth century, one of the main problem in the theory of Pfaffian equations was that of finding a canonical form for $\omega$, that is, the problem of finding a suitable change of variables, $y_{i}=\left(x_{1}, \cdots, x_{n}\right)$ so that the Pfaffian expression $\omega$ could be written in such a way as to contain the minimal number of variables. Clearly, the determination of such a canonical form coincides with the determination of the minimal number of integral equivalents of $\omega=0$ and consequently with the individuation of the integral varieties of maximal dimension.

The main results in this field were obtained by Frobenius in 1877, [18], with the introduction of two notions: the bilinear covariant (bilineare Covariante) and the class (Classe) of a Pfaffian expression.

The bilinear covariant ${ }^{6}$ of $\omega$ was defined by Frobenius as the following ex-

\footnotetext{
${ }^{4}$ In the classical literature one often finds the wording integral equivalents.

${ }^{5}$ The present-day definition of what an integral variety of a 1-form is, is quite the same, only rephrased in different language: $i: S \hookrightarrow M$ is an integral submanifold of the equation $\omega=0$ if, and only if, the pullback of $\omega, i^{*}(\omega)$, vanishes identically.

${ }^{6}$ For a detailed historical account see $[20, \S 6]$.
} 
pression:

$$
\sum_{i, j=1}^{n} a_{i j} d x_{i} \delta x_{j}, \quad \text { with } \quad a_{i j}=\frac{\partial A_{i}}{\partial x_{j}}-\frac{\partial A_{j}}{\partial x_{i}},
$$

where $d$ and $\delta$ are differentials in different directions; the word covariant indicates the crucial property according to which, if, under a change of coordinates $x_{i}^{\prime}=\phi_{i}\left(x_{k}\right), \sum A_{j} d x_{j}=\sum A_{j}^{\prime} d x_{j}^{\prime 7}$, then

$$
\sum_{i, j}^{n} a_{i j} d x_{i} \delta x_{j}=\sum_{i, j}^{n} a_{i j}^{\prime} d x_{i}^{\prime} \delta x_{j}^{\prime} .
$$

A first application of this notion was Frobenius' analytical classification theorem for Pfaffian forms ${ }^{8}$. Indeed, he considered the matrix

$$
M=\left[a_{i j}\right] \quad \text { and } \quad M^{\prime}=\left[\begin{array}{cccc}
a_{11} & \cdots & a_{n n} & A_{1} \\
\vdots & \vdots & \vdots & \vdots \\
a_{n 1} & \cdots & a_{n n} & A_{n} \\
-A_{1} & \cdots & -A_{n} & 0
\end{array}\right]
$$

and defined the class of a Pfaffian form $\omega$ as the number (invariant under arbitrary change of coordinates) $p=\frac{r k(M)+r k\left(M^{\prime}\right)}{2}$. He then demonstrated that $p$ is the minimal number of independent variables in term of which $\omega$ can be expressed. In other words, $p$ individuates the canonical form to which $\omega$ belongs: if $p=2 r$, then $\omega=y_{r+1} d y_{1}+\cdots+y_{2 r} d y_{r}$, if $p=2 r+1$, then $\omega=d y_{0}+y_{r+1} d y_{1}+\cdots+y_{2 r} d y_{r}$, under appropriate changes of coordinates.

A second application of the bilinear covariant of which Frobenius took great advantage was the so-called integrability theorem for systems of Pfaffian equations. A Pfaffian system of type

$$
\omega_{\mu}=a_{\mu 1} d x_{1}+\cdots+a_{\mu n} d x_{n}, \quad(\mu=1, \cdots, m) .
$$

was said to be completely integrable if it admits $m$ independent integrals, that is if it admits an integral variety of dimension $n-m$. Frobenius dealt with this special kind of Pfaffian systems en route for the proof of the analytical classification theorem of single Pfaffian equations. His main result was a characterization of complete integrability in terms of the properties of the bilinear covariants of (4):

\footnotetext{
${ }^{7}$ Clearly, $A_{j}^{\prime}=A_{j} \circ \phi$.

${ }^{8}$ Important results in this field were obtained by G. Darboux almost at the same time. However Darboux did not submit them for publication immediately. A paper [13] by him on Pfaff's problem appeared in 1882. For an analysis of Darboux' contribution and a comparison with Frobenius' approach, see [20, p. 420-424].
} 
Theorem 1 (Frobenius, 1877) Given the system (4) of m linearly independent Pfaffian equations, it is completely integrable if, and only if, the vanishing of all its bilinear covariants is an algebraic consequence of the system itself.

Frobenius' demonstration relied upon a result due to Clebsch which can now be interpreted as the dual counterpart of Frobenius' theorem. Indeed, in [12], Clebsch had devoted his attention to a generalization of Jacobi's theory of linear partial differential equations by introducing the notion of complete (vollständig) integrability. A system of linear partial differential equations of type

$$
A_{i}(f)=X_{i 1} \frac{\partial f}{\partial x_{1}}+\cdots+X_{i n} \frac{\partial f}{\partial x_{n}}=0, \quad(i=1, \cdots, r)
$$

was said by Clebsch to be complete if all expressions $\left(A_{i}, A_{j}\right)(f)=A_{i}\left(A_{j}(f)\right)$ $A_{j}\left(A_{i}(f)\right)$ are linear combinations (in general with non-constant coefficients) of (5). He was able to demonstrate the following:

Theorem 2 (Clebsch, 1866) If the system (5) is complete, then it admits a system of $n-r$ functionally independent solutions $f_{1}, \cdots, f_{n-r}$.

It turns out that requiring complete integrability of (4) is equivalent to the supposition that an appropriate system (actually, its dual ${ }^{9}$ ) of linear differential equations of type (5) is complete in the sense of Clebsch's definition; moreover, it should be observed that a system of integrals of (4) is also a system of solutions for (5) and viceversa.

To conclude the present introductory section, we recall that if the integrability conditions of Theorem (1) are not satisfied, then, in general, $\omega_{\mu}$ cannot be expressed as a linear combination of the total differentials of $m$ appropriate functions $f_{j},(j=1, \cdots, m)$. If this is the case, then the system (4) is said to be not completely integrable. To be precise, one should distinguish further the case in which some (although not all) of the integrability conditions are satisfied from the case in which none of them is; in the former case, one speaks of incompletely integrable systems; in the latter, of non-integrable systems. Since the study of incompletely integrable systems can be traced back to the study of non-integrable ones, we will often ignore such a distinction in the following discussion.

\footnotetext{
${ }^{9}$ It appears that Mayer was the first one to call attention over this dual connection in [25]; see also [20, p. 408-410]. In this regard, Frobenius spoke of adjungirt or zugehörig system. His characterization of duality was purely algebraic as one can see by consulting $[18, \S 13]$ or $[20$, p. 411-415]. We will see later Engel's interpretation in terms of infinitesimal transformations.
} 


\section{The state of the art in the early 1890's}

Well after the publication in 1877 of the seminal work [18] by Frobenius, the problem of finding solutions of not completely integrable Pfaffian systems remained open and almost untouched. As we have just seen, Frobenius was able to give necessary and sufficient conditions that guarantee the complete integrability of a given differential system. However, except some brief remarks ${ }^{10}$, no specific attention was paid by him to the more general problem of finding integral equivalents of not completely integrable systems of Pfaffian equations.

A common feeling of inadequacy in relation to the state of the theory of Pfaffian systems of this more general kind was frequently expressed by mathematicians in the early 1890's. For instance, Forsyth in [17] complained about the lack of new results in this realm of the theory and tried to indicate a path to be followed in order to achieve a satisfying generalization of the study of a single non-exact Pfaffian equation to systems of many equations. As in the case of a single equation, he said, it is desirable to have the integral equivalent of the system as general as possible and, in order to fulfill this aim, he individuated three different steps: i) the determination of the number of equations in the integral equivalent of a non-integrable system; ii) the deduction of some simple integral equivalent of such a system and finally, iii) the generalization of such an integral equivalent once it has been obtained. According to Forsyth, some advances had only been achieved in relation to step i) by the work [3] of Otto Biermann, who had demonstrated that the maximal dimension of the integral varieties of an unconditioned ${ }^{11}$ Pfaffian system is given by the integer part of the ratio between the number of variables and the number of equations augmented by one and that the rest of this division gives information about the degree of indeterminacy of the integral solutions. As far as the remaining two steps were concerned, Biermann's analysis had made it clear that the methods of integration at that time known (in particular the so-called Clebsch's second method) did not permit a general solution to be obtained. Forsyth's effective synthesis of the state of the art of the theory is worth quoting.

And so the solution of the problem of obtaining the integral equivalent of a simultaneous system of unconditioned Pfaffians does not appear possible by any methods at present known which are effective for the case of a single Pfaffian. It is, in fact, one of

\footnotetext{
${ }^{10}$ See $\S 20$ of [18].

${ }^{11}$ That means that no specification of the coefficients of the Pfaffian system has been made.
} 
the most general problems of the integral calculus; the discovery of its solution lies in the future. ${ }^{12}$

\section{Engel's invariants theory of Pfaffian sys- tems}

Quite similar remarks of dissatisfaction for the state of the art of the theory were expressed by F. Engel at the beginning of the first of two memoirs [15] that were dedicated to the invariants theory of Pfaffian systems and were communicated by M. A. Mayer in 1889 and in 1890 to the Sächsische Akademie der Wissenschaft in Leipzig. Engel wrote:

Die Invariantentheorie einer einzelnen Pfaff'sche Gleichung ist schon lange erledigt, dagegen bleibt für die Systeme von Pfaff'schen Gleichungen fast noch Alles zu thun. ${ }^{13}$

Engel's approach was deeply influenced by the work of his highly respected master, S. Lie. Moreover, it appears that the main concern that led him to deal with such systems of total differentials equations was their application to the theory of continuous groups of transformations. Nonetheless, beyond their applicative character, Engel's contributions are of considerable historical interest since they represented a source of inspiration for the forthcoming papers by E. von Weber and E. Cartan himself.

\subsection{Invariant correspondences}

Engel's strategy was dominated by the persistent recourse to structures invariantly connected to the given Pfaffian system. The very first example of such connected structures had been the so-called bilinear covariant of a Pfaffian expression upon which Frobenius had constantly relied in his work. Engel took up this fertile idea and generalized it, proposing the following definition: two differential systems (depending on the circumstances, a differential system can be a system of partial differential equations, a system of Pfaffian equations or a set of infinitesimal transformations) are said to be invariantly associated (invariant verknüpft) if a bijective correspondence exists between them that is preserved under arbitrary changes of coordinates. The

\footnotetext{
${ }^{12}$ See $[17, \S 185]$.

13 "The invariant theory of a single Pfaffian equation has been completed for some time; on the contrary, as far as systems of Pfaffian equations are concerned, almost everything remains to be done."
} 
knowledge of these connected structures, as in the case of a single Pfaffian equation or in the case of a complete system of Pfaffian equations examined by Frobenius, was considered by Engel quite useful since the study of their properties allowed him to get information, for example, regarding the normal form of the original Pfaffian system.

The starting point of his analysis was the observation that a reciprocal connection (Zusammenhang) exists between Pfaffian systems and systems of linear homogeneous partial differential equations of first order. According to Engel, the origin of this connection stemmed from two distinct interpretations one could ascribe to a given system of $m$ Pfaffian equations of the following form:

$$
\omega_{\mu}=\sum_{i=1}^{n} a_{\mu i}\left(x_{1} \ldots, x_{n}\right) d x_{i}=0, \quad(\mu=1, \ldots, m) .
$$

One can interpret (6), in the usual way, as a system of differential equations and, correspondingly, one can undertake the task to determine all its integral equivalent equations, that is, to determine all the equations

$$
\Phi_{1}\left(x_{1} \ldots x_{n}\right)=0, \ldots, \Phi_{q}\left(x_{1} \ldots x_{n}\right)=0
$$

such that the $2 q$ relations

$$
\Phi_{1}=0, \ldots, \Phi_{q}=0, d \Phi_{1}=0, \ldots, d \Phi_{q}=0,
$$

imply, identically, $\omega_{\mu} \equiv 0, \mu=1, \ldots, m$. On the other hand, Engel explained, one can regard the quantities $d x_{1}, \ldots, d x_{n}$ in (6) as the infinitesimal increments to which the variables $x_{1}, \ldots, x_{n}$ are subject as a consequence of the action of an infinitesimal transformation,

$$
X(f)=\sum_{j=1}^{n} \xi_{j}\left(x_{1} \ldots x_{n}\right) \frac{\partial f}{\partial x_{j}} .
$$

According to this interpretation, equations (6) define a family (Schaar) of infinitesimal transformations, namely the set of all infinitesimal transformations $X(f)$ that satisfy the following $m$ relations:

$$
\sum_{j=1}^{n} a_{\mu j} \xi_{j}=0, \quad(\mu=1, \ldots, m) .
$$

Since the rank of the matrix $A=\left[a_{\mu j}\right]$ is supposed to be maximal (and so equal to $m<n$ ), equation (7) admits $n-m$ linearly independent solutions 
$\xi_{i}^{(k)}$, for $i=1, \ldots, n$ and $k=1, \ldots, n-m$. Therefore one obtains $n-m$ linearly independent infinitesimal transformations:

$$
X_{k}(f)=\sum_{i=1}^{n} \xi_{i}^{(k)}\left(x_{1}, \ldots, x_{n}\right) \frac{\partial f}{\partial x_{i}}, \quad(k=1, \ldots, n-m) .
$$

which are the generators of the set of infinitesimal transformations that is associated to the given Pfaffian system (6). An arbitrary transformation of this set takes on the following expression:

$$
W(f)=\chi_{1}\left(x_{1}, \ldots, x_{n}\right) X_{1}(f)+\ldots+\chi_{n-m}\left(x_{1}, \ldots, x_{n}\right) X_{n-m}(f) .
$$

where the $\chi_{i},(i=1, \ldots, n-m)$ are arbitrary functions of $n$ variables.

Engel observed that this reciprocal correspondence between Pfaffian systems and sets of infinitesimal transformations is not only bijective but it is also preserved under arbitrary transformations of coordinates, so that it is, in fact, an example of invariant association. Finally, by setting all these transformations equal to zero, one obtains the following system of independent differential equations:

$$
X_{1}(f)=0, \quad \cdots, \quad X_{n-m}(f)=0 .
$$

which is also invariantly connected with (6).

This dual connection was used by Engel to build up new auxiliary Pfaffian systems which introduce remarkable simplification in the theory. The first one of these auxiliary systems is obtained as a consequence of the action of a generic infinitesimal transformation of type (8) on the Pfaffian system (6), now supposed to be rewritten in the following (aufgelöst) form ${ }^{14}$ :

$$
\Delta_{\mu}=d x_{\mu}-\sum_{k=1}^{n-m} a_{m+k, \mu} d x_{m+k}=0, \quad(\mu=1, \cdots, m) .
$$

Correspondingly, the infinitesimal transformations which are associated with it are now written as:

$$
A_{m+k}(f)=\frac{\partial f}{\partial x_{m+k}}+\sum_{\mu=1}^{m} a_{m+k, \mu} \frac{\partial f}{\partial x_{\mu}}, \quad(k=1, \ldots, n-m) .
$$

If we define $W(f)$ to be a generic transformation of type:

$$
W(f)=\sum_{k=1}^{n-m} \chi_{m+k} A_{m+k}(f)
$$

\footnotetext{
${ }^{14}$ Here and in what follows I adhere to the original notation employed by Engel.
} 
its action on (9) transforms the latter in the system:

$$
\Delta_{\mu}+\delta t\left(W \Delta_{\mu}\right)=0, \quad(\mu=1, \ldots, m)^{15}
$$

which is easily demonstrated to be equivalent and invariantly connected to the following system of Pfaffian equations:

$$
\Delta_{1}=0, \ldots, \Delta_{m}=0, A_{m+k} \Delta_{1}=0, \ldots, A_{m+k} \Delta_{m}=0, \quad(k=1, \ldots, n-m) .
$$

Finally, few manipulations give the following equivalent and simplified form written in terms of the coefficients of Frobenius' bilinear covariants:

$$
\left\{\begin{array}{c}
d x_{\mu}-\sum_{k=1}^{n-m} a_{m+k, \mu} d x_{m+k}=0 \\
\sum_{k=1}^{n-m}\left\{A_{m+k} a_{m+j, \mu}-A_{m+j} a_{m+k, \mu}\right\} d x_{m+k}=0 \\
(\mu=1, \ldots, m ; \quad j=1, \ldots, n-m) .
\end{array}\right.
$$

Engel observed that it may happen that the system (11) coincides with (9); if this is the case, then (9) is completely integrable and it admits every infinitesimal transformation (8), that is, for a generic transformation $W(f)$, $W \Delta_{\mu}=0,(\mu=1, \ldots, m)$, are a consequence of $\Delta_{\mu}=0,(\mu=1, \ldots, m)$.

In virtue of the dual correspondence between Pfaffian systems and sets of infinitesimal transformations, the system (11) can be considered as defining a set (Schaar) of infinitesimal transformations. It turns out that these transformations are precisely those transformations that leave the original Pfaffian system (9) invariant and, besides, as Engel demonstrated, that the Pfaffian system (11) is completely integrable. By using anachronistic terminology, such transformations can be called characteristic transformations and, correspondingly, the Pfaffian system (11) defining them, characteristic system.

A second differential system invariantly connected to (9) was obtained by Engel by making recourse to the following simple remark. If one considers a system of $n-m$ linear homogeneous partial differential equations of the following form:

$$
C_{k}(f)=\sum_{i=1}^{n} \beta_{i k}\left(x_{1}, \cdots, x_{n}\right) \frac{\partial f}{\partial x_{i}}=0 \quad(k=1, \cdots, n-m),
$$

and the system of equations

$$
\left\{\begin{array}{c}
\left(C_{k}, C_{j}\right)(f)=C_{k}\left(C_{j}(f)\right)-C_{j}\left(C_{k}(f)\right)=0, \\
C_{k}(f)=0, \quad(k, j=1, \cdots, n-m)
\end{array}\right.
$$

\footnotetext{
${ }^{15}$ The expression $W \Delta_{\mu}$ is what today we would call Lie derivative of $\Delta_{\mu}$ with respect to the vector field $W$. Such a denomination is very appropriate from a historical point of view. Indeed, Lie was the first one to introduce it. See for example [24, p. 529-530].
} 
then, they are invariantly associated. Since there is an adjoint Pfaffian system associated to every system of linear homogeneous partial differential equations, it is clear that two systems of Pfaffian equations which are invariantly connected correspond to (12) and (13). As a result of this, Engel stated the following:

Theorem 3 The system of Pfaffian equations which is dual to the system of partial differential equations

$$
\left\{\begin{array}{c}
A_{m+k} f=\frac{\partial f}{\partial x_{m+k}}+\sum_{\mu=1}^{m} a_{m+k, \mu} \frac{\partial f}{\partial x_{\mu}}=0 \\
\left(A_{m+k}, A_{m+j}\right) f=\sum_{\mu=1}^{m}\left(A_{m+k} a_{m+j, \mu}-A_{m+j} a_{m+k, \mu}\right) \frac{\partial f}{\partial x_{\mu}}=0 \\
(k, j=1, \cdots, n-m),
\end{array}\right.
$$

is invariantly connected to the Pfaffian system (9).

Other differential systems invariantly connected to the given Pfaffian system were obtained by Engel in the course of his researches. However, the function they fulfilled was in any case the same: to deduce from them the normal form of the considered Pfaffian system and to develop applications in the realm of the theory of continuous groups and of the theory of contact transformations as well. To name just a few concrete examples, Engel succeeded in giving a complete invariant theory of Pfaffian systems of two equations in four independent variables; furthermore, he utilized some of his results to give a simpler treatment of the problem, already faced by M. Page in [28], of the classification of all imprimitive continuous transformation groups in space in four dimensions ${ }^{16}$ and, finally, he was able to present a very clear demonstration of a theorem originally due to A. V. Bäcklund ${ }^{17}$ which gave a complete characterization of all contact transformations.

\footnotetext{
${ }^{16} \mathrm{~A}$ group of $r$ independent infinitesimal transformations in $n$ variables is said to be imprimitive if it leaves a family of $\infty^{n-q} q$-dimensional subvarieties $M_{q}$ :

$$
\phi_{1}\left(x_{1}, \ldots, x_{n}\right)=c_{1}, \quad \ldots \quad \phi_{n-q}\left(x_{1}, \ldots, x_{n}\right)=c_{n-q},
$$
}

invariant; that is, if

$$
X_{i}\left(\phi_{k}\right)=\Omega_{k i}\left(\phi_{1}, \cdots, \phi_{n-q}\right), \quad i=1, \cdots, r \quad k=1, \cdots, n-q
$$

where the $\Omega$ are some functions of $\phi_{1}, \cdots, \phi_{n-q}$; see [28, p. 297-300].

${ }^{17}$ See [4]. 


\section{Von Weber's contributions: 1898-1900}

As E. Goursat ${ }^{18}$ was once to observe, before Cartan's seminal papers [8] and [9], first rigorous results in the field of the theory of general Pfaffian systems were obtained, along with Engel, by the young mathematician Eduard Ritter von Weber (1870-1934) in a series of articles which laid the ground for the subsequent geometrical developments of Cartan's theory of exterior differential systems. Von Weber's approach was profoundly inspired to Engel's researches. Wide use of invariantly associated differential systems, frequent application of infinitesimal characteristic transformations and consistent reference to geometrical visualization were for Von Weber, as for Engel, the main technical and conceptual tools to which he had recourse to tackle the resolution of generalized Pfaffian systems.

Nonetheless, it appears that a specific motivation guided Weber's interest in his attempt to classify the large variety of Pfaffian systems, that is the hope of applying Pfaffian systems to a systematic study of general system of partial differential equations already started up by C. Méray and C. Riquier. Moreover, Weber took advantage of some of the main results of the general theory of systems of partial differential equations, namely existence theorems for the so-called passive systems ${ }^{19}$ which Weber used to demonstrate the existence of integral varieties of the given Pfaffian system and the consequent possibility of writing it in a simple normal form containing a reduced number of differentials. Finally a regular application of the theory of linear complexes and congruences in projective space has to be indicated as one of the most original technical innovation introduced by Weber into the theory ${ }^{20}$.

Weber's contributions, in which we are interested, are spread over a cer-

\footnotetext{
${ }^{18}$ See [19, p. 259].

${ }^{19}$ An explanation of this intricate notion will be given later.

${ }^{20}$ The following remarks taken from the introduction to [38] are quite enlightening. Weber wrote:

"Unsere Aufgabe lässt sich als Specialfall der allgemeinen Theorie der Differentialsysteme auffassen, wie sich auch umgekehrt die letztere, von einem andern Standpunkt aus betrachtet, der ersteren als Specialfall einordnet. Das neue Hilfsmittel jedoch, das wir bei unseren Untersuchungen verwenden und mit der Theorie der Differentialsysteme in mannigfache Beziehung setzen werden ist die Theorie der Liniencomplexe und-Congruenzen in $m$-1-dimensionalen Raum, also der Schaaren von alternirenden Bilinearformen mit $m$ Variabelnpaaren."

( "If our task can be considered as a special case of the general theory of differential systems, it is also true that, from another point of view, the latter can be regarded as a special case of the former. Yet, the new auxiliary means that we will utilize in our analysis and that will be connected in many ways to the theory of differential systems, is represented by the theory of linear complexes and congruences in $(m-1)$-dimensional space as well as by the theory of families of antisymmetric bilinear forms in $2 m$ variables").
} 
tain number of memoirs which he published between 1898 and 1900. Our attention will be mainly concentrated on [33], in which the notion of character of a Pfaffian system and that of derived system were introduced for the first time. Nevertheless, since it appears that some of his later developments may have played a role in influencing Cartan's geometrical approach to a generalization of the problem of Pfaff, a brief survey of [34], [35] and [36] will be given too.

\subsection{Character and characteristic transformations}

As Weber himself observed in the final historical remarks of [36, p. 609], since the introduction of the bilinear covariant by Frobenius and Darboux, invariantly associated structures had played a major role in the theory of Pfaffian systems. As we have seen, Engel had taken great advantage of them and had succeeded in providing some new applications of Pfaffian equations especially in the classification problem of continuous groups of transformations. Weber acknowledged the fruitfulness of this approach and tried to give it systematic basis within the context of an invariants theory of systems of Pfaffian equations.

Weber started his analysis in [33] by considering a system of $n-m$ Pfaffian equations in the following, resolved form ${ }^{21}$ :

$$
\nabla_{s}=d x_{m+s}-\sum_{i=1}^{m} a_{s i} d x_{i}=0, \quad(s=1, \cdots, n-m) .
$$

It was proved by Engel that the differential system for two independent variations of the $n$ variables $x_{1}, \cdots, x_{n}, d x_{i}$ and $\delta x_{i},(i=1, \cdots, n)$,

$$
\left\{\begin{array}{l}
d x_{m+s}=\sum a_{s i} d x_{i} ; \quad \delta x_{m+s}=\sum a_{s i} \delta x_{i}, \quad(s=1, \ldots, n-m) . \\
\sum_{k=1}^{m} \sum_{i=1}^{m} a_{i k s} d x_{i} \delta x_{k}=0,
\end{array}\right.
$$

is invariantly associated with $(15)^{22}$. As a consequence of this, Weber observed, the study of invariant quantities attached to (15) could be transferred to that of the invariants of the system (16). He defined the first of these invariants, the character $K$ of the system (15), as the rank of the matrix

$$
\left[\sum_{k=1}^{m} a_{i k s} \lambda_{k}\right] \quad(i=1, \cdots, m, s=1, \cdots, n-m),
$$

\footnotetext{
${ }^{21}$ Though it may appear bizarre, the $\nabla$ notation was that employed by Von Weber.

${ }^{22}$ As usual, it is supposed that the following relations hold: $A_{i}(f)=\frac{\partial f}{\partial x_{i}}+$ $\sum_{s=1}^{n-m} a_{s i} \frac{\partial f}{\partial x_{m+s}}$ and $a_{i k s}=-a_{k i s}=A_{i}\left(a_{s k}\right)-A_{k}\left(a_{s i}\right)$.
} 
when $x_{1}, \cdots, x_{n}$ and $\lambda_{1}, \cdots, \lambda_{m}$ assume arbitrary values.

The importance of the notion of character lay in the fact that it offered a first classification criterion for the large variety of Pfaffian systems and a measure of the difficulty degree of the problem one has to face, as it were: the greater the character, the harder is the task to undertake. If, for example, $K=0$ then system (15) is completely integrable, since clearly the coefficients of the bilinear covariants vanish identically.

Weber's attention in [33] was almost exclusively concentrated on Pfaffian systems of character one, but some important results concerning characteristic transformations were obtained for the general case too. Since characteristic transformations will also play a key role in Cartan's analysis, it seems appropriate to describe their frequent use in Weber's theory in some detail.

Already introduced by Engel, characteristic transformations are defined as the infinitesimal transformations which are dually associated with (15) and, at the same time, leave these equations invariant; that is, if we consider a generic infinitesimal transformation associated with $(15), X(f)=$ $\sum_{i=1}^{m} \xi_{i} A_{i}(f)$, this transformation is characteristic if the following identities are satisfied in virtue of equations (15):

$$
X\left(\nabla_{s}\right)=0, \quad(s=1, \cdots, n-m) .
$$

In a more explicit form, this means:

$$
X\left(\nabla_{s}\right)=d \xi_{m+s}-\sum_{i=1}^{m} X\left(a_{s i}\right) d x_{i}-\sum_{i=1}^{m} a_{s i} d \xi_{i}=0,
$$

and, consequently, we have

$$
d\left(\xi_{m+s}-\sum_{i=1}^{m} a_{s i} \xi_{i}\right)+\sum_{i=1}^{m}\left(d a_{s i} \xi_{i}-X\left(a_{s i}\right) d x_{i}\right)=0^{23},
$$

and finally, since $\xi_{m+s}=\sum_{i=1}^{m} a_{s i} \xi_{i}$ and since, as a consequence of (15), for an arbitrary function of $n$ variables, $d f=\sum_{k=1}^{m} A_{k}(f) d x_{k}$ :

$$
\sum_{k=1}^{m} \xi_{k} a_{i k s}=0, \quad(i=1, \cdots, m ; \quad s=1, \cdots, n-m) .
$$

Weber supposed that there are $h$ independent solutions $\vec{\xi}^{(i)},(i=1, \cdots, h)$ of equations (18), so that the set of infinitesimal transformations leaving

\footnotetext{
${ }^{23} \mathrm{~A}$ modern version of this formula would read as follows: $X\left(\nabla_{s}\right)=d \nabla_{s}(X)+d\left(\nabla_{s}(X)\right)$. Cartan is usually acknowledged as its first discoverer, see [23, p. 339]. However, this attribution appears to be not very accurate from a historical point of view. Indeed, it can be found already in [16, p. 415].
} 
the Pfaffian system (15) invariant is generated by the following differentials operators:

$$
X_{i}(f)=\sum_{j=1}^{m} \xi_{j}^{(i)} \frac{\partial f}{\partial x_{j}}, \quad(i=1, \cdots, h) .
$$

As already pointed out by Engel and demonstrated by Weber through a direct computation, the system of $h$ differential equations $X_{i}(f)=0$ is complete in the sense of Clebsch's definition ${ }^{24}$. Weber acknowledged the importance of such transformations and explained how they could be usefully employed to simplify the integration of the Pfaffian system under consideration. In particular, he proved the following:

Theorem 4 For the Pfaffian system (15) to be reducible, through a change of coordinates, to a system of equations in $n-h$ variables, it is necessary and sufficient that it admits $h$ (independent) characteristic infinitesimal transformations.

Indeed, if one introduces a change of coordinates in which $n-h$ variables are identified with the $n-h$ independent solutions of the complete system (19), it is easy to show that the Pfaffian system so obtained only depends upon these $n-h$ variables ${ }^{25}$.

\subsection{Pfaffian systems of character one, I}

The remaining part of the memoir [33] was devoted to a thorough analysis of a very special type of Pfaffian systems, namely those whose character is equal to one. Since Cartan would take up the same topic in 1901 by reinterpreting Weber's result in the light of his new geometrical methods based on the brand new exterior differential calculus, it appears appropriate to discuss Weber's accomplishments in order to facilitate a comparison between Cartan's and Weber's approaches.

Pfaffian systems of character one represent the simplest eventuality one can conceive, after the case of completely integrable systems. Frobenius had showed that if the system is completely integrable, then the vanishing of all its bilinear covariants is an algebraic consequence of the equations of the system itself. Instead, in the case of systems of character one the bilinear covariants reduce to a single bilinear form whose vanishing is not implied by the equations of the system itself. In other words, the following

\footnotetext{
${ }^{24}$ In modern terms, that means that the operators $X_{i},(i=1, \cdots, h)$ define an involutive distribution of tangent vector fields. See section 2 of this paper.

${ }^{25}$ For details, see [33][p. 210-211]. A modern statement of this theorem can be found in [26][p. 430].
} 
relations among the coefficients of the bilinear covariants hold: $a_{i k s}=\mu_{s} a_{i k 1}$, $(s=2, \cdots, n-m ; i, k=1, \cdots, m)$, where $\mu_{s}$ are functions of the $n$ variables $x_{1}, \cdots, x_{n}$. Weber supposed that the matrix $\left[a_{i k 1}\right],(i, k=1, \cdots, m)$ has rank equal to $2 \nu$, so that he could deduce the existence of $m-2 \nu$ linearly independent characteristic transformations

$$
X^{(k)} f=\sum_{i=1}^{n} \xi_{i}^{(k)} \frac{\partial f}{\partial x_{i}}, \quad k=1, \cdots, m-2 \nu .
$$

As already explained, the existence of such transformations was exploited to obtain a reduced form of the Pfaffian system under examination; indeed, by appropriate definition of new variables $y_{1}, \cdots, y_{\nu+1}, \cdots, y_{\nu+n-m}$, the number of differentials can be lowered to $\nu+n-m$ to give the following reduced form of (15):

$$
d y_{\nu+s}=\sum_{i=1}^{\nu} \eta_{s i} d y_{i}, \quad(s=1, \cdots, n-m)^{26}
$$

Weber's treatment of this special type of systems was marked by the definite and profitable distinction between the case in which $2 \nu=2$ and the case in which $2 \nu>2$. Let us consider in some detail the case $2 \nu>2$. The study of such systems was carried out by exploiting the existence of the so-called derived system ("das abgeleitete System von (15)") which in this case turns out to be completely integrable. Indeed, Weber considered the following system of partial differential equations (the dual counterpart of the derived system):

$$
A_{i}(f)=0, \quad B(f)=\frac{\partial f}{\partial x_{m+1}}+\sum_{s=2}^{n-m} \mu_{s} \frac{\partial f}{\partial x_{m+s}} \quad(i=1, \cdots, m)^{27}
$$

and demonstrated that it is complete in the sense of Clebsch. In fact, as $\left(A_{i} A_{k}\right)(f)=a_{i k 1} B(f) \quad(i, k=1, \cdots, m)$, all he had to show was that $\left(A_{i} B\right)(f)$ could be expressed as a linear combination (in general with nonconstant coefficients) of $A_{i}(f)$ 's and $B(f)$. Supposing ${ }^{28} m \geq 3$, from Jacobi's identity and from $\left(\left(A_{i} A_{k}\right) A_{l}\right)=\left(\sum_{s=1}^{n-m} a_{i k s} \frac{\partial f}{\partial x_{m+s}}, A_{l}\right)$, it follows that

$$
\Phi_{0 i} a_{k l 1}+\Phi_{0 k} a_{l i 1}+\Phi_{0 l} a_{i k 1}=0 \quad(i, k, l=1, \cdots, m),
$$

\footnotetext{
${ }^{26}$ It should be observed that, in general, the $\eta_{s i}$ are functions of the $n$ variables $x_{1}, \cdots, x_{n}$.

${ }^{27}$ Rember the definition of $A_{i}(f)=\frac{\partial f}{\partial x_{i}}+\sum_{s=1}^{n-m} a_{s i} \frac{\partial f}{\partial x_{m+s}} \quad(i=1, \cdots, m)$.

${ }^{28}$ The case $m=2$ is indeed trivial.
} 
where $\Phi_{0 l}=-\Phi_{l 0}=\left(B A_{l}\right)-B(f) \cdot B\left(a_{1 l}\right)$. From this one deduces that in the $(m+1) \times(m+1)$ antisymmetric matrix

$$
\left[\begin{array}{ccccc}
0 & \Phi_{01} & \Phi_{02} & \cdots & \Phi_{0 m} \\
\Phi_{10} & 0 & a_{121} & \cdots & a_{1 m 1} \\
\Phi_{20} & a_{211} & 0 & \cdots & a_{2 m 1} \\
\vdots & \vdots & \vdots & \vdots & \vdots \\
\Phi_{m 0} & a_{m 11} & a_{m 21} & \cdots & 0
\end{array}\right]
$$

all the principal minors (Hauptunterdeterminanten) of order four containing elements from the first column and the first row vanish. As a consequence of antisymmetry, all principal minors of order four vanish and consequently ${ }^{29}$, either the rank of $\left[a_{i k 1}\right]$ is two or all $\Phi_{0 l}$ vanish. Since $r k\left[a_{i k 1}\right]>2$, the only possibility is that $\Phi_{0 l}=-\Phi_{l 0}=\left(B A_{l}\right)-B(f) \cdot B\left(a_{1 l}\right) \equiv 0$ and so the system (20) is complete. From complete integrability of the system (20), Weber straightforwardly derived the complete integrability of what he called the derived Pfaffian system of (15):

$$
\nabla_{s}-\mu_{s} \nabla_{1}=0 \quad(s=2,3, \cdots, n-m) .
$$

By indicating with

$$
\left\{\begin{array}{c}
z_{2 \nu+2}\left(x_{1}, \cdots, x_{n}\right)=c_{1}, \\
z_{2 \nu+3}\left(x_{1}, \cdots, x_{n}\right)=c_{2}, \\
\vdots \\
z_{2 \nu+n-m}\left(x_{1}, \cdots, x_{n}\right)=c_{n-m-1},
\end{array}\right.
$$

its integral equivalents, he was finally able to provide a normal form for (15) given by the system

$$
\left\{\begin{array}{l}
d z_{2 \nu+1}=z_{\nu+1} d z_{1}+z_{\nu+2} d z_{2}+\cdots+z_{2 \nu} d z_{\nu} \\
d z_{2 \nu+2}=0, d z_{2 \nu+3}=0, \cdots, d z_{2 \nu+n-m}=0
\end{array}\right.
$$

where $z_{1}, \cdots, z_{2 \nu+1}$ are appropriate functions of $x_{1}, \cdots, x_{n}$. We will see later Cartan's reinterpretation of the notion of derived system. For the time being, it should be observed, as Weber did, that the derived system (23) represents an example of differential structure invariantly connected to (15) in the sense of Engel's definition. Indeed Weber's derived system (23) does coincide with the Pfaffian system introduced by Engel in Theorem 3.

\footnotetext{
${ }^{29}$ This implication holds in virtue of the antisymmetry. Remember that the rank of an antisymmetric matrix is always even and that it is equal to $r$ if, and only if all the principal minors of order $r+2$ vanish and a non vanishing principal minor of order $r$ exists.
} 


\subsection{Reducibility of a Pfaffian system to its normal form}

Although Weber's analysis in [33] can certainly be considered as remarkable progress with respect to years of relative stagnation, the results therein contained were of an unsystematic kind and often limited to very particular cases (e. g. character equal to one, as we have seen in the preceding paragraph). Over the following years, Weber tried to remedy this inconvenience and developed a more organic theory which, in principle, could be applied to Pfaffian systems of a general type. A crucial role, as we will see, was played by geometrical insight and by frequent reliance upon the theory of linear complexes and linear congruences in projective spaces.

As for Weber's results in this period, the following account is mainly based upon [34] and [35]. I will linger on some details, since in Weber's papers for the first time we encounter problems, results and technical tools of great importance for the development of Cartan's geometrical theory of exterior differential systems. When discussing Cartan's papers I will endeavour to indicate limits and relative importance of his debt to Weber.

At the beginning of [34], Weber singled out the main problem in the theory of general Pfaffian equations as the answer to the following question (indeed, a genuine generalization of the problem of Pfaff for a single total differentials equation):

Problem 1 What are the necessary and sufficient conditions for the system (15) to be reducible to the following (normal) form

$$
\sum_{s=1}^{\tau} F_{s h} d f_{s}=0, \quad(h=1, \ldots, n-m),
$$

containing only $\tau$ differentials, where $f_{1}, \cdots, f_{\tau}$ are independent functions of $x_{1}, \cdots, x_{n}$ and $\tau$ indicates an integer not smaller than $n-m$ and not greater than $n-2 ?^{30}$

As already observed by Frobenius ${ }^{31}$, if the Pfaffian system (15) admits a normal form of type (25), then the system of $\tau$ equations

$d x_{m+h}=\sum_{i=1}^{m} a_{i h} d x_{i} ; \quad d f_{1}=0, \cdots, d f_{\rho}=0 \quad(h=1, \cdots, n-m ; \rho=\tau-n+m)$

\footnotetext{
${ }^{30}$ The reason for these limitations is easily explained: if $\tau=n-1$, then one is brought back to the problem of determining 1-dimensional integral manifolds of (15); if, on the other hand, $\tau=n-m$, this means that the system (15) is completely integrable and so Frobenius' theory can be applied.

${ }^{31}$ See $[18, \S 20]$.
} 
is completely integrable. Thus, a necessary and sufficient condition for the existence of the normal form (25) is that the $n-m$ bilinear forms

$$
\Omega(d x, \delta x)=\sum_{i=1}^{m} \sum_{k=1}^{m} a_{i k s} d x_{i} \delta_{k} x, \quad(s=1, \cdots, n-m)
$$

vanish as a consequence of the following relations:

$$
\sum_{i=1}^{m} A_{i}\left(f_{k}\right) d x_{i}=0, \quad \sum_{i=1}^{m} A_{i}\left(f_{k}\right) \delta x_{i}=0 \quad(k=1, \cdots, \rho)^{32} .
$$

Weber observed that the same problem can be considered from a different and more geometrical perspective whose usefulness Cartan would thoroughly examine in his work.

If the Pfaffian system (15) can be rewritten in the normal form (25), then the equations

$$
f_{1}\left(x_{1}, \cdots, x_{n}\right)=c_{1}, f_{2}\left(x_{1}, \cdots, x_{n}\right)=c_{2}, \cdots, f_{\tau}\left(x_{1}, \cdots, x_{n}\right)=c_{\tau}
$$

represent $^{33}$ an integral equivalent of (15), that is an $(n-\tau)$-dimensional integral variety, which Weber indicated with $M_{n-\tau}$. If equations (29) are replaced by their equivalent parametric expression

$$
x_{i}=\phi_{i}\left(u_{1}, \cdots, u_{n}\right) \quad(i=1, \cdots, n ; \nu=n-\tau),
$$

then these functions are solutions of a first order differential system, to be indicated with the symbol $S_{\nu}$, which takes on the following form ${ }^{34}$ :

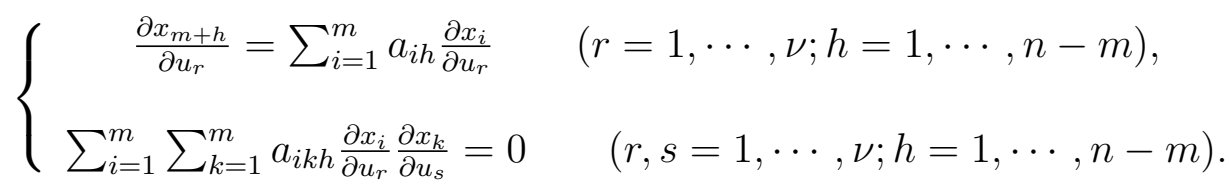

\footnotetext{
${ }^{32}$ This is a good point at which the following important observation should be made: in the classical literature no conceptual and notational distinctions were made between base elements of what we would nowadays call cotangent space and the components of tangent vectors. If one cannot resist the temptation to restore such a distinction, it should be observed that in formulas (27) and (28) the $d x_{i}$ 's and the $\delta x_{i}$ 's have to be regarded as components of tangent vectors whereas in formula (26) the $d x_{i}$ 's and $d f_{k}$ 's are indeed to be considered as elements of a cotangent space. Clearly, this comes as no surprise as far as the theory lacks a formal definition of what a differential form is. A little bit more surprising will be the discovery that even Cartan's theory was affected by this "flaw".

${ }^{33}$ For arbitrary values of the $c_{i}$ 's.

${ }^{34}$ The second group of equations can be easily deduced from the first one simply by differentiating with respect to $u_{1}, \cdots, u_{\nu}$ and remembering that $\frac{\partial^{2} x_{i}}{\partial u_{r} \partial u_{s}}=\frac{\partial^{2} x_{i}}{\partial u_{s} \partial u_{r}}$. For a detailed verification of this very simple statement, see e. g. [2, p. 110-112]
} 
Thus, if the differential system $S_{\nu}$ is such that there is a $M_{n-\tau}$ integral variety through every point $\left(x_{1}^{0}, x_{2}^{0}, \cdots, x_{n}^{0}\right)$ of a certain domain of the whole variety $M_{n}$, then the Pfaffian system admits the normal form (25). In this way, problem (1) was traced back to the analysis of the conditions guaranteeing the existence of solutions of the differential system $S_{\nu}$.

It was at this very point that Weber made recourse to the theory of the so-called general systems of partial differential equations developed, among others, by C. Méray and C. Riquier ${ }^{35}$. As we will see, a crucial role was played by the notion of passivity (or involution) which assures, under regularity conditions, the existence of integrals of the system itself.

If one adds to the system $S_{\nu}$ all the equations that can be obtained from $S_{\nu}$ through repeated derivations (finite in numbers), either of the following two eventualities must occur: either a contradiction, that is a relation among the variables $x_{1}, \cdots, x_{n}$ only is produced or a differential system is obtained such that, by solving it with respect to certain partial derivatives, it can be put in the so-called canonical passive form, $\Sigma .{ }^{36}$ If this is the case and if $\nu$ constants $u_{1}^{0}, \cdots, u_{\nu}^{0}$ are arbitrarily chosen, the system $S_{\nu}$ admits a unique solution $x_{k}\left(u_{1}, \cdots, u_{\nu}\right),(k=1, \cdots, n)$ with the property that the parametric quantities valued in $u_{1}^{0}, \cdots, u_{\nu}^{0}$ assume the initial values

$$
x_{1}^{0}, \cdots, x_{n}^{0}, \cdots,\left(\frac{\partial^{i+k+\cdots+l} x_{h}}{\partial u_{1}^{i} \partial u_{2}^{k} \cdots \partial u_{\nu}^{l}}\right)_{0} \cdots,
$$

\footnotetext{
${ }^{35}$ For a historical account of the theory, Riquier's remarks in the preface to [30] can be consulted.

${ }^{36}$ I will not insist on detail. For further details, see Riquier's papers [29] and his treatise [30] or Weber's encyclopaedia article [37] on partial differential equations. I will give just few remarks. The expression canonical form means a system (to be indicated with $\Sigma$ ) of equations of type:
}

$$
\frac{\partial^{\alpha_{1}+\alpha_{2}+\cdots+\alpha_{\nu}} x_{i}}{\partial u_{1}^{\alpha_{1}} \partial u_{2}^{\alpha_{2}} \cdots \partial u_{\nu}^{\alpha_{\nu}}}=\phi_{i, \alpha_{1}, \cdots, \alpha_{\nu}}\left(x_{1}, \cdots, x_{\nu}, \cdots, \frac{\partial^{\beta_{1}+\cdots+\beta_{\nu}} x_{k}}{\partial u_{1}^{\beta_{1}} \cdots \partial u_{\nu}^{\beta_{\nu}}}\right)
$$

which satisfies the following requirements: i) No derivative making its appearance in the left side of (32) is contained in the right side. ii) for every derivative $\frac{\partial^{\beta_{1}+\cdots+\beta_{\nu}} x_{k}}{\partial u_{1}^{\beta_{1} \ldots \partial u_{\nu}^{\beta_{\nu}}}}$ contained in the expression for $\phi_{i, \alpha_{1}, \cdots, \alpha_{\nu}}$, is $\sum_{j=1}^{\nu} \beta_{j} \leq \sum_{j=1}^{\nu} \alpha_{j}$; if, in particular, $\sum_{j=1}^{\nu} \beta_{j}=\sum_{j=1}^{\nu} \alpha_{j}$ then $k \leq i$; if even $k=i$ then the first non-vanishing number in the series of differences $\beta_{1}-\alpha_{1}, \beta_{2}-\alpha_{2}, \cdots$ is required to be positive.

On the other hand, passivity coincides with the following hypothesis: if the partial derivatives contained in the left side of the equations in $\Sigma$ and all the other deduced from them through repeated derivations with respect to $u_{1}, \cdots, u_{\nu}$ are called principal, and the remaining ones, along with the dependent variables $x_{1}, \cdots, x_{n}$, are called parametric quantities, then it is required that from $\Sigma$ and from equations deduced by $\Sigma$ through differentiation, every principal derivative is required to be expressed in terms of the parametric quantities in a unique way. 
provided that the right-hand sides of equations composing $\Sigma$ are sufficiently regular and also provided that the $n$ power series (the sum being extended to all parametric derivatives):

$$
\sum\left(\frac{\partial^{i+k+\cdots+l} x_{k}}{\partial u_{1}^{i} \cdots u_{\nu}^{l}}\right)_{u_{i}=u_{i}^{0}}\left(u_{1}-u_{1}^{0}\right)^{i} \cdots\left(u_{\nu}-u_{\nu}^{0}\right)
$$

converge in a certain region of $C^{n}$. In this way, the admissibility of a normal form of type (25) and the consequent existence of $n-\tau$-dimensional integral varieties of the Pfaffian system was characterized by Weber in terms of the possibility to write the differential system $S_{\nu}$ in a canonical passive (involutive) form. Moreover, as he observed, to establish whether such an eventuality was fulfilled or not, reduced, at least in principle, to a simple procedure consisting of differentiations and eliminations to be operated upon $S_{\nu}$.

At this point, Weber made an interesting observation that was destined to assume a role of outstanding importance in Cartan's theory. Motivated by the possibility of developing fruitful applications in the realm of the theory of general partial differential equations, he introduced further hypotheses which guarantee the existence of integral varieties of increasing dimensions. Indeed, he explained, if one supposes that the system $S_{\nu}$ can be put in a canonical passive form simply by solving it with respect to certain derivatives $\frac{\partial x_{i}}{\partial u_{\nu}}$ and furthermore, one supposes that from $S_{\nu}$ and from equations obtained from it by differentiation and elimination, no relation among the variables $x_{i}, \frac{\partial x_{i}}{\partial u_{1}}, \cdots, \frac{\partial x_{i}}{\partial u_{\nu}}, \quad(i=1, \cdots, n)$, already contained in $S_{\nu-1}$, can be deduced, and so on for the systems $S_{\nu-1}, \cdots, S_{1}$, then every 1-dimensional integral variety of (15) $M_{1}$ belongs at least to one 2-dimensional integral variety $M_{2}$, etc. and, finally, every $\nu$-1-dimensional integral variety $M_{\nu-1}$ belongs at least to one $\nu$-dimensional integral variety $M_{\nu}$.

Thus, Weber arrived at the statement of what I will refer to as the second problem of his theory of Pfaffian systems.

Problem 2 What are the necessary and sufficient conditions that have to be satisfied so that every 1-dimensional integral variety $M_{1}$ of (15) belongs at least to one 2-dimensional integral variety $M_{2}$ etc., and so that every $\nu$-1-dimensional integral variety $M_{\nu-1}$ belongs at least to one $\nu$-dimensional integral variety $M_{\nu}$ ?

Clearly, both for Problem 1 and Problem 2, the aim was that of expressing such conditions by means of algebraic and differential relations among the coefficients $a_{s i},(s=1, \cdots, n-m ; i=1, \cdots, m)$ only.

For instance, it turns out that a necessary condition to be fulfilled for the system (15) to possess $\nu$-dimensional integral varieties, $M_{\nu}$ (Problem 1), 
is the existence of a system of linearly independent functions $\eta_{i}^{(s)},(i=$ $1, \cdots, m ; s=1, \cdots, \nu)$ satisfying the following bilinear equations:

$$
\sum_{i=1}^{m} \sum_{k=1}^{m} a_{i k h} \eta_{i}^{(r)} \eta_{k}^{(s)}=0, \quad(r, s=1, \cdots, \nu ; h=1, \cdots, n-m) .
$$

A detailed account of Weber's achievements over this point would go beyond our present purposes; we will include just a few observations.

His analysis was mainly based on the theory of bilinear forms and, more precisely, on the classification of linear complexes and linear congruences in $(m-1)$-dimensional ${ }^{37}$ projective spaces. This should come as no surprise, since the coefficients of the bilinear covariants, $a_{i k s},(i, k=1, \cdots, m ; s=$ $1, \cdots, n-m)$ were interpreted by Weber geometrically as defining a system of linear complexes, equal in number to the character $K$ of (15):

$$
\sum_{i=1}^{m} \sum_{k=1}^{m} a_{i k s} \eta_{i} \xi_{k}=0, \quad(s=1, \cdots, K) .
$$

In this geometrical context, Weber identified the characteristic transformations, $X_{i}(f)=\sum_{j=1}^{m} \xi_{j}^{(i)} \frac{\partial f}{\partial x_{j}},(i=1, \cdots, h)$, as those for which the components $\xi_{j}$ represent the singular points of the congruence consisting of all the straight lines belonging to complexes (35).

Weber was able to give a detailed analysis of Pfaffian systems with $m=$ $3,4,5,6$ but, ultimately, despite his hopes ${ }^{38}$, his approach did not succeed in providing a general theory of unlimited validity. Nevertheless, he should be acknowledged for opening a new phase in the studies of general Pfaffian systems, assessing some of the problems to be considered relevant and also for introducing useful technical tools which were destined to outlive his theory itself and to be reinterpreted in the light of Cartan's exterior differential calculus.

\section{The foundations of the exterior differential calculus}

After the composition of his doctoral thesis [5] where he gave a rigorous and complete treatment of the classification problem of finite-dimensional,

\footnotetext{
${ }^{37}$ The number $m$, that is the difference between the number of variables and the number of equations of which the system (15) consists, was called by Weber die Stufe and it was considered by him, along with the character, as a measure of the difficulties one has to face in tackling the study of a given system of Pfaffian equations. See [38, p. 387].

${ }^{38}$ See, [38, p. 388].
} 
semisimple, complex Lie algebras already started up and developed by Killing, for some years Cartan devoted himself to applications of the theoretical results contained therein. The theory of partial differential equations appears to be one of the main fields of his interest. This emerges quite clearly, for example, from the reading of a dense memoir, [6], dedicated to the theory of those systems of partial differential equations whose solutions depend only upon arbitrary constants and such that they admit a continuous group of transformations.

Cartan's work [7] on Pfaffian forms and more specifically on the problem of Pfaff was part of this interest. Indeed, as Lie had demonstrated, the integration of partial differential equations and the integration of Pfaffian forms were considered as equivalent formulations of the same problem.

V. Katz [22] and T. Hawkins [20] have already given a full and authoritative account of the large part of the material contained in [7]. For this reason we will limit ourselves to recall the main notions which will be useful for the rest of our discussion.

Cartan organized his treatment in a deductive way by first presenting a full set of definitions and conventions. He started up by giving a symbolic definition of what a differential expression in $n$ variables is; this was defined as a homogenous expression built up by means of a finite number of additions and multiplications of the $n$ differentials $d x_{1}, \cdots, d x_{n}$ as well as of certain coefficients which are functions of $x_{1}, \cdots, x_{n}$. In such a way, a Pfaffian expression was defined as a differential expression of degree one of type: $A_{1} d x_{1}+\cdots+A_{n} d x_{n}$; a differential form of degree two was given, for example, by $A_{1} d x_{2} \wedge d x_{1}+A_{2} d x_{3} \wedge d x_{2}{ }^{39}$.

A very important notion of Cartan's new calculus was the exterior multiplication between two differential expressions ${ }^{40}$. Cartan himself observed that already in 1896 he had realized that the variables change formulas in multiple integrals could be easily derived by submitting the differentials under the integration sign to appropriate laws of calculation which coincide with Grassmann's exterior calculus. By developing such an intuition, in 1899 he was able to present convincing arguments to justify such rules, which relied upon the idea of the value of a differential form.

To this end, Cartan considered a differential expression $\omega$ of degree $h$ and then supposed that the $n$ involved variables are functions of $h$ arbitrary parameters $\left(\alpha_{1}, \cdots, \alpha_{h}\right)$. By indicating with $\left(\beta_{1}, \cdots, \beta_{h}\right)$ one of the $h$ ! permutations of the parameters $\alpha_{1}, \cdots, \alpha_{h}$, Cartan associate to it the value that $\omega$ assumes

\footnotetext{
${ }^{39}$ Cartan did not employ the wedge product symbol $\wedge$.

${ }^{40}$ One can find the germs of this crucial notion already in some works on integral invariants by Poincaré. See [22, p. 322] and [27, p. 69] for the relevant bibliography.
} 
when the differentials occupying the $i^{\text {th }}(i=1, \cdots, h)$ position are replaced by the corresponding derivative of $x$ with respect to $\beta_{i}$. By attributing to such a quantity the sign + or - depending on the parity of the permutation considered and then by summing over all $h$ ! permutations, Cartan finally obtained what he called the value of the differential expression. For example, the value of the differential form $A_{1} d x_{2} \wedge d x_{1}+A_{2} d x_{3} \wedge d x_{2}$ is:

$$
A_{1} \frac{\partial x_{2}}{\partial \alpha_{1}} \frac{\partial x_{1}}{\partial \alpha_{2}}+A_{2} \frac{\partial x_{3}}{\partial \alpha_{1}} \frac{\partial x_{3}}{\partial \alpha_{2}}-A_{1} \frac{\partial x_{2}}{\partial \alpha_{2}} \frac{\partial x_{1}}{\partial \alpha_{1}}-A_{2} \frac{\partial x_{3}}{\partial \alpha_{2}} \frac{\partial x_{2}}{\partial \alpha_{1}} .
$$

At this point, Cartan defined two differential expressions of degree $h$ to be equivalent if their value is the same independently from the choice of parameters $\alpha_{1}, \cdots, \alpha_{h}$. In this a way he was able to establish Grassmann's well-known rules for the multiplication to be interpreted, in Cartan's view, as equalities between equivalence classes of exterior differential forms. For example, one has $d x_{1} \wedge d x_{2}=-d x_{2} \wedge d x_{1}$ or $d x_{4} \wedge d x_{4}=0$, as it is easy to see by calculating the values of the differential expressions appearing in the equations.

A second crucial novelty of Cartan's theory was the exterior derivative of a given Pfaffian expression ${ }^{41}$ which he explicitly connected with Frobenius' and Darboux's notion of bilinear covariant. Cartan's definition reads as follows. Given a Pfaffian form of type $A_{1} d x_{1}+\cdots+A_{n} d x_{n}$, its derived expression was the form of degree two:

$$
\omega^{\prime}=d A_{1} \wedge d x_{1}+\cdots+d A_{n} \wedge d x_{n}{ }^{42} .
$$

The invariant character of such derivative was then established upon reliance of the notion of value by observing that if $\bar{\omega}$ indicates the expression of $\omega$ with respect to a new set of coordinates $y_{i}(\vec{x})$ then the differential forms of degree two $\overline{\omega^{\prime}}$ and $(\bar{\omega})^{\prime}$ are equivalent in the sense specified above.

On the basis of such a new calculus, Cartan not only was able to reformulate all the known results of the theory of Pfaffian equations, including Frobenius' analytical classification theorem ${ }^{43}$, but he also succeeded in obtaining new remarkable achievements concerning the resolution of systems (particularly relevant for the theory of partial differential equations of first order ) consisting of a single Pfaffian equation and a certain number of finite relations ${ }^{44}$.

\footnotetext{
${ }^{41}$ The definition was generalized to enclose derivatives of differential forms of degree greater than one in [9, p. 243].

${ }^{42}$ The notation $d \omega$ was introduced by Kähler in $[21$, p. 6].

${ }^{43}$ See section 2 above.

${ }^{44}$ See $[7$, Chapter V].
} 


\section{Cartan's theory of general Pfaffian systems}

After laying the foundations of his new exterior differential calculus, Cartan devoted himself to the study of not completely integrable systems of Pfaffian equations. As for Weber, in accordance with the motivations laying at the basis of [7], it appears that the main reason for this was to be found in the wide applications of Pfaffian expressions to the theory of partial differential equations.

Cartan started his analysis in [8] by recalling Biermann's ${ }^{45}$ efforts to determine the maximal dimension and the degree of indeterminacy of integral varieties of unconditioned Pfaffian systems. In this respect, Cartan complained about the lack of rigorous (not generic) and systematic results, and emphasized the urgency to provide the theory with solid theoretical grounds in order to remedy this unsatisfactory state of affairs.

As already observed by Hawkins ${ }^{46}$, a key role was played by the notion of the bilinear covariant which, as we have seen, Cartan interpreted as the first exterior derivative of a Pfaffian form. However, one should not forget that the use of such a notion was not Cartan's prerogative since, as we have seen, its employment was quite frequent among other mathematicians too. Instead, what characterizes Cartan's approach with respect to his contemporaries was the ubiquitous recourse to geometrical insight, and the foundational role of his exterior differential calculus which introduced considerable simplifications in the theory.

\subsection{Geometrical representation}

Apparently, Lie had been the first one to attribute a geometrical interpretation to the system of Pfaffian equations (15) in the context of his synthetic approach to differential equations. Engel took it from Lie and profitably applied it to his researches in [15] by writing:

Man kann mit dem Systeme (15) auch eine anschauliche Vorstellung verbinden. Durch die Gleichungen (15) wird nämlich jedem Punkte des Raumes $x_{1}, \cdots, x_{n}$ ein ebenes Bündel von $\infty^{m-1}$ Fortschreitungsrichtungen: $d x_{1}: d x_{2}: \cdots: d x_{n}$ zugeordnet. Denkt man sich in jedem Punkte des Raumes das zugehörige Bündel von Fortschreitungsrichtungen, so erhält man eine Figur, welche das genaue geometrische Bild des Systems (15) ist ${ }^{47}$.

\footnotetext{
${ }^{45}$ See section 3 above.

${ }^{46}$ See $[20$, p. 430]

47 "We can attribute to the system (15) also an illustrative representation. Indeed,
} 
Weber himself took great advantage of such a geometrical representation, and even widened it by introducing for the first time (September 1900, [35]) the notion of element of a Pfaffian system as the set of directions tangent to the integral variety of the system itself.

Clearly inspired by this longstanding tradition, Cartan opened his analysis in [8] by emphasizing the importance of geometrical representation in the problems he was about to deal with. Let us consider a system of Pfaffian equations of the following type:

$$
\left\{\begin{array}{c}
\omega_{1}=a_{11} d x_{1}+\cdots+a_{1 r} d x_{r}=0 \\
\omega_{2}=a_{21} d x_{1}+\cdots+a_{2 r} d x_{r}=0 \\
\vdots \\
\omega_{s}=a_{s 1} d x_{1}+\cdots+a_{s r} d x_{r}=0
\end{array}\right.
$$

Cartan supposed that $n$ out of the $r$ variables should be regarded as independent, so that the remaining $r-n$ could be expressed as functions of them. In this way, a $n$-dimensional variety $M_{n}$ of the $r$-dimensional total manifold ${ }^{48}$ was defined. Then, Cartan observed, the system (36) can be thought of geometrically as prescribing those conditions that have to be satisfied by the differentials $d x_{1}, \cdots, d x_{r}$ when one considers an arbitrary displacement on $M_{n}$. Furthermore, since the differentials $d x_{i}$ can be assimilated ${ }^{49}$ to direction parameters of the tangent lines to the variety $M_{n}$, the system (36) can be interpreted by saying that, as a consequence of (36), these tangent lines belong to a certain linear variety (multiplicité plane) which depends upon the point considered. Hence, the problem of finding $n$-dimensional integral varieties of (36) was traced back to the following:

A chaque point de l'espace on fait correspondre une multiplicité plane passant per ce point; déterminer une multiplicité à $n$ dimensions $M_{n}$, telle qu'en chacun des ses points toutes les tangentes à cette multiplicité soient situées dans la multiplicité plane correspondente à ce point ${ }^{50}$.

through the equations (15), to every point of the space $x_{1}, \cdots, x_{n}$ the corresponding plane bundle of directions: $d x_{1}: d x_{2}: \cdots: d x_{n}$, is associated. If in every point of the space the corresponding bundle of directions is considered then one obtains a figure which is the exact image of the system (15)."

${ }^{48} \mathrm{I}$ will designate with the expression total manifold the set of all $r$-uple of type $\left(x_{1}, \cdots, x_{r}\right)$.

${ }^{49}$ Again, no distinction between base elements of cotangent spaces and components of tangent vectors was made.

50 "We associate to every point of the space a linear variety passing through this point; then we determine a $n$-dimensional variety $M_{n}$, such that in its every point all the tangents 
After posing the problem in precise geometrical terms, Cartan defined a linear element (élément linéaire) to be the set consisting of a point and a straight line passing through this point which can be denoted with $(\vec{x}, d \vec{x})$. Then, he specified a linear element to be integral if the differentials $d x_{1}, \cdots, d x_{n}$, regarded as direction parameters of the tangent line of the linear element, satisfy the systems of linear equations obtained from (36) after evaluating the coefficients $a_{i j},(i=1, \cdots, s ; j=1, \cdots, r)$, in $\vec{x}$. He finally arrived at the the following proposition which characterized the integral varieties of (36).

Proposition 1 For a variety to be integral is necessary and sufficient that every linear element of it be integral.

The notion of linear element was soon generalized to a greater number of dimensions. Indeed, a $p$-dimensional element was defined to be the couple consisting of a point and a linear variety passing through this point. Cartan indicated it with the symbol $E_{p}$. It was clear that every $p$-dimensional element $E_{p}$ of an integral variety $M_{n}$ (necessarily, $p \leq n$ ) consists of integral linear elements; however, as already observed by Weber ${ }^{51}, E_{p}$ has to satisfy further conditions which, in general ${ }^{52}$, are not algebraically implied by (36); they are the relations obtained by requiring the vanishing of all bilinear expressions

$$
\left\{\begin{array}{c}
\omega_{1}^{\prime}=\sum_{i, k=1}^{r}\left(\frac{\partial a_{1 i}}{\partial x_{k}}-\frac{\partial a_{1 k}}{\partial x_{i}}\right)\left(d x_{i} \delta x_{k}-d x_{k} \delta x_{i}\right), \\
\vdots \\
\omega_{s}^{\prime}=\sum_{i, k=1}^{r}\left(\frac{\partial a_{s i}}{\partial x_{k}}-\frac{\partial a_{s k}}{\partial x_{i}}\right)\left(d x_{i} \delta x_{k}-d x_{k} \delta x_{i}\right),
\end{array}\right.
$$

where $d \vec{x}$ and $\delta \vec{x}$ are arbitrary integral linear elements belonging to $E_{p}$. Thus, Cartan arrived at the crucial definition of integral element of more than one dimension:

to the variety belong to the linear variety corresponding to this point." Cartan's formulation of the integration problem can be translated as follows. Consider the exterior ideal $\mathcal{I}$, simply generated by the 1 -forms $\omega_{1}, \cdots, \omega_{s}$. To find integral submanifolds of $\mathcal{I}$ is equivalent to the problem of determining integral submanifolds of the distribution of vector fields which is dual to $\mathcal{I}$. If we indicate this distribution with $\mathcal{V}=\left\{v_{1}, \cdots, v_{r-s}\right\}$, the vector space $\left.\mathcal{V}\right|_{x}$ is precisely the linear variety to which Cartan referred. Indeed, for a submanifold $M_{n}$ to be an integral variety of $\mathcal{V}$ is necessary and sufficient that $\left.\left.T M_{n}\right|_{x} \subset \mathcal{V}\right|_{x}, \forall x \in M_{n}$.

${ }^{51}$ Here we are referring to Weber's remarks according to which one has to consider in the differential system $S_{\nu}$, together with equations of type $\frac{\partial x_{m+h}}{\partial u_{r}}=\sum a_{i h} \frac{\partial x_{i}}{\partial u_{r}}$, also the equations obtained by the them through differentiation.

${ }^{52}$ Unless the system (36) is completely integrable. 
Appelons élément intégral à 2,3,... dimensions un élément formé d'éléments linéaires intégraux et tel, de plus, que deux quelconques d'entre eux satisfassent au system (37). ${ }^{53}$

Then he defined two integral linear elements $d \vec{x}, \delta \vec{x}$ to be associated or in involution if all bilinear expressions (37) vanish. As a consequence of this, the definition of integral element could be rephrased as follows:

\section{Un élément integral à $2,3, \cdots$ dimensions est un élément formé d'éléments linéaires intégraux associés deux à deux. ${ }^{54}$}

Once more, Cartan found it useful to emphasize his geometrical approach by observing that if the quantities $d x_{i} \delta x_{k}-d x_{k} \delta x_{i}$ are regarded as Plücker's coordinates of a straight line then the bilinear relations (37) can be interpreted as defining linear complexes in projective spaces; in this respect, he often insisted upon the possibility of yielding a thorough classification of all Pfaffian systems on the basis of the classification of linear complexes themselves.

It is somehow surprising that Cartan never mentioned Weber's valuable researches $^{55}$ in this field, namely Weber's frequent recourse to the theory of linear complexes and bilinear forms. It is true that they had been published only a year before the publication of Cartan's epoch-making article [8] and he thus might not have had the opportunity of studying them carefully or even of reading them at all. Nevertheless, it is of considerable historical interest that many ideas fully developed by Cartan could already be found in Weber's work.

We will see later that for the crucial notion of a Pfaffian system in involution (closely related to Problem 2 of Weber's theory) too, no mention of Weber's analysis was made by Cartan. Once more, it is probable that Cartan was not aware of Weber's work, and that he introduced involutive systems borrowing them directly from the theory of general systems of partial differential equations as developed by Méray, Riquier and É. Delassus.

\footnotetext{
53 "An element consisting of linear integral elements is said to be an integral element of dimension $2,3, \ldots$ if every two linear integral elements satisfy (37)."

In modern terms, Cartan's definition of integral element of (36) can be translated as follows: a $p$-dimensional integral element of (36) is a subspace $\left.E_{p} \subset T M_{r}\right|_{x}$ such that:

$$
<\omega_{j} ; v_{i}>=0 \wedge<d \omega_{j} ; v_{i}, v_{k}>=0 \quad \forall v_{i}, v_{k} \in E_{p}, \quad(j=1, \cdots, s) .
$$

54 "A linear integral element of dimension $2,3, \cdots$ is an element consisting of linear integral elements which are associated pairwise".

${ }^{55}$ The only work by Weber, among those here considered, explicitly cited by Cartan both in [8] and [9] was [33].
} 


\subsection{Cauchy's first theorem}

What Cartan called Cauchy's first problem was the following:

Étant donnée une multiplicité intégrale à $p$ dimensions $M_{p}$ d'un système d'equations aux différentielles totales, faire passer par $M_{p}$ une multiplicité intégrale à $p+1$ dimensions $M_{p+1} \cdot{ }^{56}$

Its relevance can hardly be overestimated. Indeed, it turns out that the problem of integration of (36) can be solved by a step by step procedure consisting of determining integral varieties of increasing dimensions. Clearly, a necessary condition for the existence of a $(p+1)$-dimensional integral variety $M_{p+1}$ passing through $M_{p}$ is that every $p$-dimensional integral element $E_{p}$ of $M_{p}$ is contained at least in one $(p+1)$-dimensional integral element $E_{p+1}$. However this condition is not sufficient. To guarantee the existence of such integral varieties one has to require more, i.e. that at least a $(p+1)$-dimensional $E_{p+1}$ integral element passes through every $p$-dimensional integral element of the space.

Before moving on to the resolution of Cauchy's first problem, Cartan observed, it is useful to state a few geometrical remarks on the structure of the integral elements $E_{p+1}$. If $E_{p}$ is supposed to be generated by $p$ linearly independent vectors, i.e. $E_{p}=<e_{1}, \cdots, e_{p}>$ then an integral element $E_{p+1} \supset E_{p}$ can be defined by adding a linear element $e$ to $E_{p}$, which is linearly independent from $E_{p}$; clearly, one requires both that $e$ is integral and that $e$ is in involution with $e_{i}, \forall i=1, \cdots, p$.

The $(p+1)$-dimensional element $E_{p+1}$ containing $E_{p}$ depends on $r-p$ homogeneous parameters ${ }^{57}$, and the equations expressing that $E_{p+1}$ is integral are linear with respect to these parameters. Let us suppose that these equations reduce in number to $r-p-u-1$ (with $s \geq 0$ ), then at least one integral element $E_{p+1}$ passes through every integral element $E_{p}$. In particular if $u=0$ then such a $E_{p+1}$ is unique. In general, these integral elements build up an infinite family depending on $u$ arbitrary constants. It may happen that in particular cases the degree of indeterminacy is greater than $u$; in this eventuality, Cartan said that $E_{p}$ is a singular element, otherwise $E_{p}$ was said to be regular $^{58}$. Besides, the notion of singularity was easily transferred to integral

\footnotetext{
56 "Given a p-dimensional integral variety $M_{p}$ of a system of total differentials equations, to determine $a(p+1)$-dimensional integral variety $M_{p+1}$ passing through $M_{p} . "$

${ }^{57}$ The numbers of effective parameters is $r-p-1$; in general, every $q$-dimensional element $E_{q}$ containing a $p$-dimensional element $E_{p}$ depends on $(q-p)(r-q)$ effective parameters. See Goursat's proof in $[19, \S 81]$.

${ }^{58} \mathrm{~A}$ more clear explanation of Cartan's notion of regularity will be given in the next section in terms of the so-called characteristic integers.
} 
varieties by defining them to be singular when all their integral elements are singular in the specified sense.

At this point Cartan was ready to face the solution of Cauchy's first problem proving what he called Cauchy's first theorem. This is a crucial point in the whole theory of exterior differential systems; indeed, it can be considered as the gist of what is nowadays known as Cartan-Kähler theorem, since one can find in it the proof of the inductive step that is crucial for assessing the existence of varieties "integrating" integral elements. As is well-known, the validity of the theorem is limited to the class of analytic Pfaffian system. For this reason we will suppose, as Cartan explicitly did, that the coefficients of (36) are analytic functions of $x$.

Theorem 5 (Cauchy's first theorem) Suppose that every p-dimensional, regular, integral element passes through at least one $(p+1)$-dimensional integral elements $E_{p+1}$; then, given a non-singular p-dimensional integral variety of (36), $M_{p}, a(p+1)$-dimensional integral variety $M_{p+1}$ exists which passes through $M_{p}$. More precisely, if $\infty^{u}(p+1)$-dimensional integral elements passes through every regular, integral element $E_{p}$, then many integral varieties $M_{p+1}$ exist which depend upon u arbitrary functions.

In order to offer a general idea of how it works it will be enough to limit ourselves to the following remarks.

Cartan's starting point was to translate the geometrical content of the statement into an analytic form. To this end he considered on $M_{p}$ a $p$-dimensional regular integral element $E_{p}^{0}$ that he supposed to have its centre in a fixed point $P^{0}=\left(x_{1}^{0}, \cdots, x_{r}^{0}\right)$, of $M_{p}$. In an appropriate open subset containing $P^{0}, M_{p}$ can be represented by $r-p$ analytic functions expressing, for instance, the variables $x_{p+1}, \cdots, x_{r}$ in terms of $x_{1}, \cdots, x_{p}$. Furthermore, the linear equations (with constant coefficients) defining the integral element $E_{p}^{0}$ are solvable with respect to the differentials $d x_{p+1}, \cdots, d x_{r}$. Then if a $(p+1)$-dimensional integral element $E_{p+1}^{0}$ passing through $E_{p}^{0}$ is considered, the $r-p-1$ equations defining it are solvable with respect to the $r-p-1$ differentials $d x_{p+2}, \cdots, d x_{r}$, say. Thus, a $(p+1)$-dimensional integral variety $M_{p+1}$ admitting $E_{p+1}^{0}$ will be expressed, in an appropriate neighbourhood of $P^{0}$, by $r-p-1$ analytic functions, $x_{p+2}, \cdots, x_{r}$ of $x_{1}, \cdots, x_{p+1}$.

At this point Cartan introduced some simplification in the notation: $x_{p+1}$ was replaced by $x$ and $x_{p+2}, \cdots, x_{r}$ by $z_{1}, \cdots, z_{m}$ (clearly, $m=r-p-1$ ). As a consequence of this, the equations for $M_{p}$ (in a neighbourhood of $P^{0}$ ) 
can be written as follows:

$$
\left\{\begin{array}{c}
x=\phi\left(x_{1}, \cdots, x_{p}\right), \\
z_{1}=\phi_{1}\left(x_{1}, \cdots, x_{p}\right) \\
\vdots \\
z_{m}=\phi_{m}\left(x_{1}, \cdots, x_{p}\right),
\end{array}\right.
$$

while the variety $M_{p+1}$ can be expressed (in a neighborhood of $P^{0}$ ) by:

$$
z_{j}=z_{j}\left(x, x_{1}, \cdots, x_{p+1}\right), \quad(j=1, \cdots, m=r-p-1) .
$$

If $x$ is replaced by $x-\phi\left(x_{1}, \cdots, x_{p}\right)$ then (38) can be rewritten as

$$
\left\{\begin{array}{l}
x=0 \\
z_{j}=\phi_{j}\left(x_{1}, \cdots, x_{p}\right), \quad(j=1, \cdots, m) .
\end{array}\right.
$$

Consequently, the condition that $M_{p}$ passes through $M_{p+1}$ requires that:

$$
z_{j}\left(0, x_{1}, \cdots, x_{p}\right)=\phi_{j}\left(x_{1}, \cdots, x_{p}\right) \quad(j=1, \cdots, m) .
$$

Now, the problem consists of writing down the differential equations for the $r-p-1$ unknown functions that have to be satisfied for $M_{p+1}$ to be an integral variety of (36). To this end, it is first necessary to consider the generic $(p+1)$ dimensional element (having its centre in a neighborhood of $P^{0}$ ) consisting of the linear elements that one obtains when infinitesimal increments are attributed to every single variable $x, x_{1}, \cdots, x_{p}$. Their direction parameters are given by the following table:

$$
\begin{array}{cc}
e: & \frac{d x}{1}=\frac{d x_{1}}{0}=\cdots=\frac{d x_{p}}{0}=\frac{d z_{1}}{\frac{\partial z_{1}}{\partial x}}=\cdots=\frac{d z_{m}}{\frac{\partial z_{m}}{\partial x},} \\
e_{1}: & \frac{d x}{0}=\frac{d x_{1}}{1}=\cdots=\frac{d x_{p}}{0}=\frac{d z_{1}}{\frac{\partial z_{1}}{\partial x_{1}}}=\cdots=\frac{d z_{m}}{\frac{\partial z_{m}}{\partial x_{1}}}, \\
\vdots & \vdots \\
e_{p}: & \frac{d x}{0}=\frac{d x_{1}}{0}=\cdots=\frac{d x_{p}}{1}=\frac{d z_{1}}{\frac{\partial z_{1}}{\partial x_{p}}}=\cdots=\frac{d z_{m}}{\frac{\partial m_{m}}{\partial x_{p}}} .
\end{array}
$$

Then one requires that this $(p+1)$-dimensional element is indeed an integral element; that is, one requires i) that every linear element is integral and ii) that the linear integral elements are pairwise in involution. Cartan observed that it is useful to separate such a system of differential equations into two groups: the first one (I) assures that the element $E_{p}=\left\langle e_{1}, \cdots, e_{p}\right\rangle$ is integral, the second one (II) that the linear element $e$ is integral and in involution with $E_{p}$. It is easily seen that (I) does not contain the derivatives 
$\frac{\partial z_{1}}{\partial x}, \cdots, \frac{\partial z_{m}}{\partial x}$ while the second system of equations (II) is linear with respect to such derivatives. At this point the regularity assumptions come into play: essentially, the fact that the indeterminacy degree of $E_{p+1}$ is minimal guarantees the possibility to rewrite the system (II) in a Cauchy-Kovalevskaya form where $m-u$ derivatives are expressed in terms of an equal number of analytic functions:

$$
\left\{\begin{array}{c}
\frac{\partial z_{1}}{\partial x}=\Phi_{1}\left(x, x_{i}, z_{k}, \frac{\partial z_{k}}{\partial x_{j}}, \frac{\partial z_{m-u+1}}{\partial x}, \cdots, \frac{\partial z_{m}}{\partial x}\right) \\
\vdots \\
\frac{\partial z_{m-u}}{\partial x}=\Phi_{m-u}\left(x, x_{i}, z_{k}, \frac{\partial z_{k}}{\partial x_{j}}, \frac{\partial z_{m-u+1}}{\partial x}, \cdots, \frac{\partial z_{m}}{\partial x}\right) .
\end{array}\right.
$$

The theory of the systems of partial differential equations of this particular kind guarantees the existence of holomorphic solutions in a neighbourhood of $P^{0}$ depending on $u$ arbitrary ${ }^{59}$ functions $z_{m-u+1}, \cdots, z_{m}$, such that for $x=0$ they reduce to $\phi_{1}, \cdots, \phi_{m}$.

Cartan's final step consisted of proving that the solution so obtained indeed represents an $(p+1)$-dimensional integral variety of the system (36), that is, it satisfies both system (I) and (II). First, he proved that every solution of the Cauchy-Kovalevskaya system satisfies systems (I) and (II) for $x=0$. Then he demonstrated that every solution satisfying (I) and (II) for a generic $x$ also satisfies (I) and (II) for every infitesimally close point $x+\delta x$, showing, in such a way, that the theorem is true for every point within an appropriate neighbourhood of $P^{0}$.

As we have seen, Weber had been the first one to set the problem of the integration of general Pfaffian systems in terms of the existence of integral varieties of increasing dimension. Yet he was unable to yield a systematic analysis of the conditions whose determination was the object of Problem 2 of his theory. For his part, Cartan considered the determination of chains of integral varieties of increasing dimension as the gist of the integration procedure of general Pfaffian systems. To this end, he introduced the following definition of Pfaffian system in involution. A Pfaffian system (36) was said by him to be in involution if at least one 2-dimensional integral variety $M_{2}$ passes through each integral curve $M_{1}$, at least one 3-dimensional integral variety $M_{3}$ passes through each 2-dimensional integral variety $M_{2}$, etc., and finally at least one $g$-dimensional integral variety $M_{g}$ passes through each $(g-1)$-dimensional integral variety $M_{g-1}{ }^{60}$. Now, from the first Cauchy's theorem it follows that a sufficient and necessary condition for the system

\footnotetext{
${ }^{59}$ Clearly, for $x=0$ they have to reduce to $\phi_{m-u+1}, \cdots, \phi_{m}$.

${ }^{60}$ The number $g$ was called by Cartan the genre of the system (36); it is defined as the maximal dimension of regular integral varieties of (36). In the next section, we will
} 
(36) to be in involution is that every regular 1-dimensional integral element $E_{1}$ belongs at least to one 2-dimensional integral element $E_{2}$, every regular 2-dimensional integral element $E_{2}$ belongs at least to one 3-dimensional integral element, etc., and finally, every regular $(g-1)$-dimensional integral element $E_{g-1}$ belongs at least to one $g$-dimensional integral element $E_{g}$.

In a such a way Cartan was able to provide a complete answer to Weber's Problem 2 by obtaining at the same time an answer to Problem 1 since the determination of the number $g$ coincides with the determination of integral (regular) varieties of maximal dimension.

Some remarks on the historical origin of the notion of involutive systems of Pfaffian equations are in order here. Weber was the first, to my knowledge, to draw attention on the relation between the general theory of canonical passive (involutive) systems of partial differential equations and the existence of chains of integral varieties of (36) of increasing dimensions. Thus, it would be natural to trace back Cartan's notion of involution to Weber's works on general Pfaffian systems, namely to [34]. However, I cannot produce any evidence testifying that Cartan derived from Weber the inspiration for his researches over this point. On the contrary, Cartan's recollection contained in [11][p. 28-29] seems to support the possibility that he developed such a notion independently of Weber. In fact he recognized that his own notion of involution was analogous to the one introduced, among the others, by Méray and Riquier in the context of the theory of general systems of partial differential equations.

Lastly, it seems that an important influence on Cartan was exerted by the work of Étienne Delassus, namely by [14], in which, for the first time, the study of solutions of general systems of partial differential equations had been traced back to the study of Cauchy-Kovalevskaya systems. In particular, Delassus had shown that the whole integration procedure could be reduced to successive integrations of Cauchy-Kovalevskaya systems in an increasing number of independent variables. From this point of view, Cartan's achievements can be seen as a translation and a development of Delassus' results in the geometric, coordinates-independent language of exterior differential forms.

\subsection{Genre and characters}

Cauchy's first theorem highlights the need to proceed toward a detailed geometrical analysis of the properties of integral elements. In particular, as

see how the existence of such an integer can be deduced from the so-called characteristic integers of $(36)$. 
we have just seen, Cartan was interested in studying the conditions guaranteeing the existence of integral elements of increasing dimensions. His procedure can be summarized as follows: let us consider a $p$-dimensional integral element $E_{p}$ of a certain point $x$; we can think of it as being generated by $p$ linearly independent linear integral elements reciprocally in involution: $E_{p}=<e_{1}, \cdots, e_{p}>$. To construct an integral element $E_{p+1}$ containing $E_{p}$, as we already know, one has to add to $E_{p}$ an integral linear element $e$ independent from $E_{p}$ as well as in involution with $e_{i},(i=1, \cdots, p)^{61}$. If, as Cartan would do in [9], we introduce the linear variety ${ }^{62} H\left(E_{p}\right)$, the polar element ${ }^{63}$ of $E_{p}$, of all integral linear elements in involution with $E_{p}$, we can rephrase the preceding sentence by saying that $E_{p+1}=<E_{p}, e>$, with $e \in H\left(E_{p}\right)$ and $e \notin E_{p}$.

To characterize the structure of such polar elements as well as to obtain information on the degree of indeterminacy of integral elements, Cartan introduced a sequence of integers, which we will indicate with $\tilde{r}_{i}$, to be defined as follows:

$$
\operatorname{dim} H\left(E_{p}\right)=\tilde{r}_{p+1}+p+1 .
$$

Geometrically, this means that the polar element $H\left(E_{p}\right)$ is generated by the $p$ base vectors of $E_{p}$ and by $\tilde{r}_{p+1}+1$ linear integral elements, $e_{0}, e_{1}, \cdots, e_{\tilde{r}_{p+1}}$. As a consequence of this, the $(p+1)$-dimensional integral elements $E_{p+1}$ passing through $E_{p}$ depend on $\tilde{r}_{p+1}$ parameters or, as Cartan expressed himself, $\infty^{\tilde{r}_{p+1}}$ integral elements $E_{p+1}$ pass through $E_{p}$.

Although Cartan was not very explicit, it is clear that the coefficients $\tilde{r}_{p}$ depend not only on the point $x$ to which the $E_{p}$ 's belong but also on the choice of the basis of the tangent space in $x$ to the integral variety (indeed, even on the ordering of such a basis $)^{64}$. Thus, to be rigorous, he should have written $\tilde{r}_{p}\left(x ; e_{1}, \cdots, e_{p-1}\right)$ instead of $\tilde{r}_{p}$. In fact, it appears that the coefficients $r_{p}$ effectively introduced by Cartan, the so-called characteristic integers, should be interpreted as the minimum values of $\tilde{r}_{p}\left(x ; e_{1}, \cdots, e_{p-1}\right)$ when $x$ varies over $M_{r}$ and the $e_{i}$ 's $(i=1, \cdots, p-1)$ vary over $\left.T M_{r}\right|_{x}$ :

$$
r_{p}=\operatorname{Min}\left\{\tilde{r}_{p}\left(x, e_{1}, \cdots, e_{p-1}\right)\left|x \in M_{r}, e_{i} \in T M_{r}\right|_{x}\right\} .
$$

\footnotetext{
${ }^{61}$ Remember that $e$ is in involution with $E_{p}=<e_{1}, \cdots, e_{p}>$ if, and only if $d \omega_{k}\left(e, e_{i}\right)=$ $0,(k=1, \cdots, s ; i=1, \cdots, p)$.

${ }^{62}$ The fact that $H\left(E_{p}\right)$ is a linear variety is a consequence of the bilinearity of (37). It should be observed that, although consisting of linear integral elements, in general, $H\left(E_{p}\right)$ is not an integral element. The reason for this is due to the fact that two linear integral elements in involution with a third are not necessarily in involution between themselves. See $[8$, p. 250].

${ }^{63}$ To my knowledge, this denomination was first introduced by Cartan when discussing Pfaffian systems of character one in $[9, \S 18]$.

${ }^{64}$ See $[26$, p. $450-454]$.
} 
This lack of notational precision was justified, in Cartan's view, by the necessity to focus his analysis ${ }^{65}$ on the so-called regular integral elements, i.e. on those integral elements for which $\tilde{r}_{p}=r_{p}$. More precisely, according to Cartan's definition, a $p$-dimensional integral element $E_{p}$ is regular if, and only if, $\tilde{r}_{p+1}=r_{p+1}^{66}$, or, in another words, when its polar element has minimal dimension. As a consequence of this, in that which follows, as Cartan did, we will limit our attention to non-singular (i.e. regular) integral elements and for this reason we will ignore the distinction between $r_{p}$ and $\tilde{r}_{p}$.

From these preliminary remarks, Cartan moved on to demonstrate certain arithmetical relations among the characteristic integers which turn out to be very useful for the following discussion.

A first result is that the integers $r_{p}$ decrease when the index $p$ increases. Indeed let us consider a regular integral element $E_{p}$ and a regular integral element $E_{p-1}$ contained therein; since every linear integral element in involution with $E_{p}$ is, a fortiori, in involution with $E_{p-1} \subset E_{p}$, we have $H\left(E_{p}\right) \subset H\left(E_{p-1}\right)$ and thus, $r_{p} \geq r_{p+1}+1$.

From this, it follows that the succession of integers $\left\{r_{p}\right\}$ is decreasing and that an integer $g$ exists such that $r_{g+1}=-1$. Therefore, the polar space $H\left(E_{g}\right)$ does coincide with $E_{g}$ (supposed to be regular) and no $(g+1)$-dimensional integral element $E_{g+1}$ passes through $E_{g}$. The integer $g$ was called by Cartan the genre of the differential system (36).

Another chain of inequalities gives information on the differences among three consecutive characteristic integers:

$$
r_{p}-r_{p+1} \geq r_{p+1}-r_{p+2} \quad(p \leq g-2) .
$$

As for the preceding inequality, its demonstration relies on geometric considerations concerning the polar spaces, $H\left(E_{p-1}\right), H\left(E_{p}\right)$ and $H\left(E_{p+1}\right)$.

From this and from $r_{g-1}-r_{g}-1 \geq r_{g}$ Cartan finally deduced the following fundamental chain of inequalities:

$$
r-r_{1}-1 \geq r_{1}-r_{2}-1 \geq \cdots \geq r_{g-1}-r_{g}-1 \geq r_{g} .
$$

The numbers present in such inequalities assume a great importance in the theory. As the characteristic integers $r_{p}$, they are invariants of the system (36) with respect to arbitrary changes of coordinates ${ }^{67}$ and provide a useful

\footnotetext{
${ }^{65}$ As we will see later, characteristic elements are a major exception.

${ }^{66}$ It should be observed that this is different from the notion of regularity given for example by Olver in [26, p. 456].

${ }^{67} \mathrm{Such}$ an invariance property is essentially due to the covariance of the exterior derivative. See [9, p. 236-237].
} 
tool for the classification of general Pfaffian systems. Cartan indicated them with $s_{1}, \cdots, s_{g}$, namely ${ }^{68}$ :

$$
\left\{\begin{array}{c}
s_{1}=r_{1}-r_{2}-1 \\
\vdots \\
s_{g-1}=r_{g-1}-r_{g}-1 \\
s_{g}=r_{g} .
\end{array}\right.
$$

Cartan observed that the first of these integers, $s_{1}$, had already been introduced by Weber under the denomination of character of the system (36). By generalizing such a notion to the subsequent integers, Cartan spoke of second, third, etc. character, respectively. As we have seen, Weber had introduced the character $s_{1}$ in a purely algebraic manner (except for the subsequent interpretation in terms of linear complexes) as the number of linearly independent relations built up with the bilinear covariants of the Pfaffian system. It is easy to demonstrate that Cartan's definition coincides with that of Weber. Indeed, it is sufficient to observe that, according to Cartan's definition, $s_{1}$ is the number of linearly independent equations which one has to add to (36) in order to obtain the polar element of a (regular) linear integral element, $E_{1}$.

The relevance of such integers was clarified by the possibility of determining the most general (regular) integral variety $M_{g}$ of (36), by repeated application of the so-called Cauchy's first theorem. More precisely, Cartan was able to yield a full characterization of the indeterminacy degree of the solutions of (36) by demonstrating the following

Theorem 6 (Cauchy's second theorem) Given a Pfaffian system of $s$ linearly independent equations in $r$ variables, let us indicate with $g$ its genre and with $s_{1}, \cdots, s_{g}$ its characters. Then, the $r$ variables can be divided into $g+2$ groups:

$$
\begin{array}{cccc}
x_{1}, & x_{2}, & \cdots, & x_{g} ; \\
z_{1}, & z_{2}, & \cdots, & z_{s} ; \\
z_{1}^{(1)}, & z_{2}^{(1)}, & \cdots, & z_{s_{1}}^{(1)} \\
\vdots & \vdots & \vdots & \vdots \\
z_{1}^{(g)}, & z_{2}^{(g)}, & \cdots, & z_{s_{g}}^{(g)} .
\end{array}
$$

such that on the most general integral variety $M_{g}$ the variables $x_{1}, x_{2}, \cdots, x_{g}$ can be regarded as independent and, in a neighborhood of a regular point

\footnotetext{
${ }^{68}$ The first integer $r-r_{1}-1$ is ignored since it is easily demonstrated to be equal to $s$, the number of linear independent Pfaffian equations of the system. $s$ was sometimes called the zero-th character of (36), for example in [2].
} 
$\left(x_{1}^{0}, \cdots, x_{g}^{0}\right), M_{g}$ is determined by the following specification of initial conditions: on $M_{g}, z_{1}^{(g)}, z_{2}^{(g)}, \cdots, z_{s_{g}}^{(g)}$ reduce to $s_{g}$ arbitrary functions of $x_{1}, \cdots, x_{g}$; for $x_{g}=x_{g}^{0}$, the $z_{1}^{(g-1)}, \cdots, z_{s_{g-1}}^{(g-1)}$ reduce to $s_{g-1}$ arbitrary functions of $x_{1}, \cdots, x_{g-1}$; etc.; for $x_{g}=x_{g}^{0}, \cdots, x_{2}=x_{2}^{0}$ the $z_{1}^{(1)}, z_{2}^{(1)}, \cdots, z_{s_{1}}^{(1)}$ reduce to $s_{1}$ functions of $x_{1}$; finally, for $x_{g}=x_{g}^{0}, \cdots, x_{2}=x_{2}^{0}, x_{1}=x_{1}^{0}$, the $z_{1}, \cdots, z_{g}$ reduce to $s$ arbitrary constants.

As Cartan observed, the theorem includes the results already obtained by Biermann ${ }^{69}$ in the realm of unconditioned Pfaffian systems. Clearly, Cartan's achievements were far more general, rigorous and complete. Furthermore, the characterization of the indeterminacy of the integral solutions agreed with analogous results obtained by Delassus in [14] in his researches on general systems of partial differential equations. Nevertheless, Cartan claimed the superiority of his new approach through exterior differential forms since it had the advantage of being independent of a particular choice of coordinates.

\subsection{Characteristic elements}

Thus far, we have dealt with regular integral elements. However, singular integral elements play an important role too. Following Engel, Weber had introduced characteristic transformations as those infinitesimal transformations which are dual to the Pfaffian equations of the system and leave the system invariant. For his part, Cartan introduced what he called characteristic elements by observing that in some cases the differential equations of the Cauchy-Kovalevskaya system determining the integral variety $M_{p+1}$ passing through a given integral variety $M_{p}$ assume a particularly simple form which greatly simplifies their integration. By using the notation of section (7.2), Cartan considered the eventuality in which such equations do not depend upon the derivatives $\frac{\partial z_{i}}{\partial x_{k}},(i=1, \cdots, r-p-1 ; k=1, \cdots, p)$, that is the case in which the Cauchy-Kovalevskaya system is independent of the linear integral elements $e_{1}, \cdots, e_{p}$ generating the integral element $E_{p}$. As a consequence of this, the partial derivatives $\frac{\partial z_{1}}{\partial x}, \cdots, \frac{\partial z_{r-p-1}}{\partial x}$ define a linear integral element $e$ which depends only on the point considered and is as well in involution with all the integral elements $E_{p}$ passing through this point. If this is the case, in every point of the space ${ }^{70}$ a linear integral element exists that is in involution with every linear integral element drawn from this point. Cartan called such linear integral elements characteristic $^{71}$. Clearly, a linear

\footnotetext{
${ }^{69}$ See section 3 of this paper.

${ }^{70}$ We actually should limit ourselves to some open subset of the space.

${ }^{71}$ The denomination stemmed from the theory of partial differential equations, namely from the theory of Cauchy's characteristics. The connection between Cartan's character-
} 
integral characteristic element is also singular, since $\infty^{r_{1}-1}$ integral elements $E_{2}$ pass through it.

As Weber had already observed, the importance of characteristic elements ${ }^{72}$ lay in the possibility to exploit their existence to simplify the integration of the Pfaffian system under examination. Indeed, after defining the characteristic Pfaffian system as the system of total differentials equation determining characteristic elements, he was able to reformulate Weber's Theorem 4 in the following way:

Theorem 7 The minimal number of variables upon which, by means of a change of variables, the coefficients and the differentials of a given Pfaffian system can depend is equal to the number of linear independent equations of the characteristic system; these variables are given by the integration of such a system.

As for Weber's analysis, a crucial point in Cartan's treatment was the fact that the characteristic system is completely integrable. However, whereas Weber had established such a property by making recourse to what we called characteristic transformations and then relying upon Clebsch's theorem on complete systems of linear partial differential equations, Cartan deliberately avoided such expedients and managed to demonstrate the complete integrability of the characteristic system by using differential forms only. Within few months, he was able to propose two different demonstrations. The first one contained in [8, p. 302-305] consisted of a step by step procedure which relied on the basic property according to which a Pfaffian system of $r-1$ equations in $r$ variables is necessarily completely integrable. The second, contained in [9, p. 248-249], was instead presented as a more direct application of the symbolic calculus with exterior differential forms that Cartan had developed in [7].

It should be noticed that, as we will observe in the next section too, Cartan's refusal to utilize infinitesimal transformations was by no means casual. We suggest that his need to avoid any recourse to them was due to the project of developing an approach to continuous Lie's groups purely in terms of Pfaffian forms without any use of infinitesimal transformations which, according to Cartan's view ${ }^{73}$, did not represent an appropriate technical tool to deal with the structural theory of infinite dimensional continuous groups of transformations.

istic elements and Cauchy's characteristics is explained very clearly in [2, p. 180-182].

${ }^{72}$ Actually, as Engel did, he spoke of transformations leaving the Pfaffian system invariant.

${ }^{73}$ See, for instance, [10, Chap. 2]. 


\subsection{Pfaffian systems of character one, II}

The present section will be devoted to a discussion of Cartan's analysis contained in [9] of this special type of Pfaffian systems with the aim to draw a comparison between Weber's and Cartan's approaches. Whereas Weber's treatment was almost entirely based on analytical and algebraic considerations, Cartan carried out his analysis in geometrical terms, heavily relying, as in his approach to characteristic elements, on the new properties of his exterior differential calculus.

Since the beginning of his discussion, Cartan affirmed that his analysis of Pfaffian systems of character one does not bring to any new results with respect to Weber's paper [33]. Nevertheless, he set out to reinterpret Weber's achievements in order to yield a concrete application of the principles of his theory.

Let us begin with Cartan's deduction of the so-called derived system of (36). Contrary to Weber's analysis which had had recourse to infinitesimal transformations (indeed, the dual counterpart of differential expressions) to deduce, via Theorem 3, the existence of the system (23) invariantly connected to the Pfaffian system under examination, Cartan first defined the notion of congruence between two differential forms ${ }^{74}$, and then he introduced the derived system in the following way.

He considered a Pfaffian system of $s$ independent equations, $\omega_{i}=0,(i=$ $1, \cdots, s)$, in $r$ variables and he introduced $r-s$ Pfaffian forms $\bar{\omega}_{j},(j=$ $1, \cdots, r-s)$ such that $\left\{\omega_{i}, \bar{\omega}_{j}\right\}(i=1, \cdots, s ; j=1, \cdots, r-s)$ are $n$ independent Pfaffian forms ${ }^{75}$. As a consequence of this, the $s$ bilinear covariants of the system can be written as

$$
\omega_{i}^{\prime} \equiv \sum_{j, k=1}^{n-s} A_{i j k} \bar{\omega}_{j} \wedge \bar{\omega}_{k}=\Omega_{i}, \quad\left(\bmod \omega_{1}, \omega_{2}, \cdots, \omega_{s}\right), \quad(i=1, \cdots, s) .
$$

Now, in general, the differential forms $\Omega_{i}$ are not independent; if, for example:

$$
l_{1} \Omega_{1}+l_{2} \Omega_{2}+\cdots+l_{s} \Omega_{s}=0^{76},
$$

then one has:

$$
\left(l_{1} \omega_{1}+l_{2} \omega_{2}+\cdots+l_{s} \omega_{s}\right)^{\prime} \equiv 0, \quad\left(\bmod \omega_{1}, \omega_{2}, \cdots, \omega_{s}\right) .
$$

\footnotetext{
${ }^{74}$ If $\Omega$ and $\Pi$ designate two differential forms with the same degree and $\omega_{1}, \cdots, \omega_{p}$ designate $p$ homogeneous differential forms with degree less or, at most, equal to that of $\Omega$ (and $\Pi$ ), then Cartan defined $\Omega$ and $\Pi$ to be congruent module $\omega_{1}, \cdots, \omega_{p}$ if $p$ differential forms $\chi_{1}, \cdots, \chi_{p}$ exist such that: $\Omega=\Pi+\omega_{1} \wedge \chi_{1}+\cdots+\omega_{p} \wedge \chi_{p}$.

${ }^{75}$ In modern terms, one can say that $\left\{\omega_{i}, \bar{\omega}_{j}\right\}(i=1, \cdots, s ; j=1, \cdots, r-s)$ define a coframe.

${ }^{76}$ Here and in what follows, $l_{i},(i=1, \cdots, s)$ indicate $s$ arbitrary functions of $x_{1}, \cdots, x_{r}$.
} 
In such a way, Cartan demonstrated that appropriate linear combinations of the Pfaffian equations of the system (36) exist such that every couple of integral elements of (36) is in involution with respect to them. He then considered all the equations of type $l_{1} \omega_{1}+l_{2} \omega_{2}+\cdots+l_{s} \omega_{s}=0$, and built up what he called the derived system of (36). It is clear that Cartan's definition was a generalization of that given by Weber which was limited to systems of character one. Furthermore, it is important to emphasize the fact that the introduction of derived systems was brought about by Cartan purely in terms of exterior differential forms without any recourse to operations with vector fields (infinitesimal transformations, in his wording). To this end, an important role may have been played by the remark, already implicit in Engel's and Weber's work, that exterior differentiation could be considered in a certain sense as the dual counterpart of the Lie-bracketing operation between two infinitesimal transformations ${ }^{77}$.

Specializing his discussion to systems of character one, in accordance with Weber's results, Cartan was able to show that in this case the derived system of (36) consists of the $s-1$ equations:

$$
\omega_{i}-l_{i} \omega_{1}=0 \quad(i=2, \cdots, s) .
$$

and, consequently, that the equations of (36) could be chosen in such a way that:

$$
\omega_{2}^{\prime} \equiv \omega_{3}^{\prime} \equiv \cdots \equiv \omega_{s}^{\prime} \equiv 0 \quad\left(\bmod \omega_{1}, \cdots, \omega_{s}\right)
$$

Before turning to a detailed study of the derived system and exploiting its properties to integrate the Pfaffian system under examination, it is necessary, Cartan observed, to examine carefully the geometric properties of the (unique) linear complex associated to (36). A first problem to be solved is the determination of the maximal dimension of (regular) integral elements and consequently the maximal dimension of (regular) integral varieties. In the light of Weber's results, one may expect that characteristic elements play a role of strategic importance and, indeed, it turns out that this is the case also for Cartan's treatment of the subject.

Cartan started by considering the linear variety of all linear integral elements of (36); he indicated it with $H_{\rho}$, where $\rho$ designates its dimension that is, $\rho=r-s$. He supposed that $\sigma$ is the dimension of the greatest characteristic element $\epsilon_{\sigma}$ and then considered a linear integral element $E_{1} \notin \epsilon_{\sigma}$; since $E_{1}$ is supposed to be regular and the character of (36) is assumed to be equal to 1 , its polar element $H_{\rho-1}$, is a linear variety of dimension $\rho-1$. Now, with

\footnotetext{
${ }^{77}$ For a detailed discussion of the notion of derived system with special emphasis on duality, see [31, p. 24-26]
} 
respect to the linear integral elements of $H_{\rho-1}$, Cartan observed, a characteristic element $\epsilon_{\sigma+1}$ of dimension $\sigma+1$ exists such that $\epsilon_{\sigma+1}=<\epsilon_{\sigma}, E_{1}>^{78}$. It turns out, as Cartan demonstrated in full detail, that $\epsilon_{\sigma+1}$ is the greatest characteristic element with respect to $H_{\rho-1}$. At this point, he considered another linear integral element $E_{1}^{\prime}$ not belonging to $\epsilon_{\sigma+1}$; thus, the linear elements of $H_{\rho-1}$ in involution with $E_{1}^{\prime}$ generate a linear variety $H_{\rho-2}$ whose greatest characteristic element $\epsilon_{\sigma+2}$, Cartan demonstrated, can be described as the linear variety $<\epsilon_{\sigma}, E_{1}, E_{1}^{\prime}>$. Iterating the same process an appropriate number of times, one finally arrives at a (necessarily integral) element $H_{\rho-\nu}{ }^{79}$ which coincides with its characteristic element $\epsilon_{\sigma+\nu}$. The number $\nu$ is obtained by equating the dimension of $H_{r-\nu}$ with that of $\epsilon_{\sigma+\nu}$; thus:

$$
\rho-\sigma=2 \nu
$$

Consequently, since $H_{\rho-\nu}$ is one of the integral elements of (36) of maximal dimension, the genre of the Pfaffian system is $\rho-\nu^{80}$.

Now, from the previous geometrical construction of maximal integral elements, it follows that the bilinear covariant $\omega_{1}^{\prime}$ must be expressed in terms of $2 \nu$ independent Pfaffian forms, so that:

$$
\omega_{1}^{\prime}=\bar{\omega}_{1} \wedge \bar{\omega}_{\nu+1}+\cdots \bar{\omega}_{\nu} \wedge \bar{\omega}_{2 \nu} \quad\left(\bmod \omega_{1}, \cdots, \omega_{s}\right) .
$$

This formula is the starting point for the subsequent analytical study of integral varieties of systems of character one.

To emphasize the novelty of Cartan's technical tools with respect to those utilized by Weber, let us consider his demonstration of the theorem according to which if $\nu>1$ then the derived system of (36) is completely integrable. Cartan supposed that the Pfaffian forms of (36) are chosen in such a way that its derived system can be written as:

$$
\omega_{2}=\omega_{3}=\cdots=\omega_{s}=0 .
$$

From Cartan's definition of derived system it follows that:

$$
\omega_{2}^{\prime} \equiv \omega_{3}^{\prime} \equiv \cdots=\omega_{s}^{\prime} \equiv 0 \quad\left(\bmod \omega_{1}, \cdots, \omega_{s}\right) .
$$

\footnotetext{
${ }^{78}$ The fact that $\epsilon_{\sigma+1}$ is characteristic with respect to $H_{\rho-1}$ means that $e \in \epsilon_{\sigma+1}$ if, and only if $\omega_{i}(e)=0,(i=1, \cdots, s)$ and $\omega_{1}^{\prime}\left(e, e^{\prime}\right)=0 \forall e^{\prime} \in H_{\rho-1}$. The statement that $<\epsilon_{\sigma}, E_{1}>$ is characteristic with respect to $H_{\rho-1}$ should now be clear if one recalls the definition of polar element.

${ }^{79}$ Cartan indicated with $h$ what here is indicated with $\nu$. The change in notation is aimed at facilitating the comparison with [33].

${ }^{80}$ This is in accordance with Weber's normal form (24) for the case in which $\nu>1$.
} 
As a consequence of $\omega_{2}^{\prime} \equiv 0,\left(\bmod \omega_{1}, \cdots, \omega_{s}\right)$, one has:

$$
\omega_{2}^{\prime} \equiv \omega_{1} \wedge \chi,\left(\bmod \omega_{2}, \cdots, \omega_{s}\right),
$$

where $\chi$ is a form of degree one which depends upon $\omega_{1}, \bar{\omega}_{1}, \cdots, \bar{\omega}_{2 \nu}{ }^{81}$. By calculating the derivative of the last congruence, one obtains that

$$
\omega_{1}^{\prime} \wedge \chi-\chi^{\prime} \wedge \omega_{1} \equiv 0 \quad\left(\bmod \omega_{2}, \cdots, \omega_{s} ; \omega_{1}^{\prime}, \cdots, \omega_{s}^{\prime}\right)
$$

and, consequently, that $\omega_{1}^{\prime} \wedge \chi \equiv 0,\left(\bmod \omega_{1}, \cdots, \omega_{s}\right)$. This is equivalent to:

$$
\omega_{1}^{\prime}=\chi \wedge \pi+\omega_{1} \wedge \pi_{1}+\cdots+\omega_{s} \wedge \pi_{s}
$$

for appropriate forms of degree one, $\pi, \pi_{1}, \cdots, \pi_{s}$. Now, unless $\chi \equiv 0\left(\bmod \omega_{1}\right)$, the integral elements of (36) that satisfy $\chi=0$ and $\pi=0$ would be characteristic elements and consequently the number of equations of the characteristic system would be $s+2$. However, in such an eventuality, we would have $\nu=1$ which contradicts the hypothesis of the theorem to be demonstrated. Thus, the only possibility is that $\chi \equiv 0\left(\bmod \omega_{1}\right)$. As result of this, from $(48)$ we obtain: $\omega_{2}^{\prime} \equiv 0\left(\bmod \omega_{2}, \cdots, \omega_{s}\right)$, and, after repeating the same reasoning for $\omega_{3}, \cdots, \omega_{s}$, we finally deduce that :

$$
\omega_{2}^{\prime} \equiv \omega_{2}^{\prime} \equiv \cdots \equiv \omega_{s}^{\prime} \equiv 0 \quad\left(\bmod \omega_{2}, \cdots, \omega_{s}\right)
$$

which, according to Cartan's reformulation of Frobenius' theorem ${ }^{82}$, implies the complete integrability of the derived system of (36).

\section{Final remarks}

Contrary to prevalent opinion, far from being the result of the work of an isolated mathematical genius, Cartan's theory of exterior differential systems (later on generalized by Kähler in [21] to differential systems of any degree), was deeply rooted in the historical context of the late nineteenth century theory of partial differential equations. Indeed, as we have seen, his achievements were situated at the intersection of two closely related strands of research: the theory of not completely integrable systems of Pfaffian equations as developed by Engel and Weber, and the theory of general systems of partial differential equations that was the main focus of attention of Méray, Riquier and Delassus, among others. Cartan's great merit was to reinterpret

\footnotetext{
${ }^{81}$ This is due to the fact that, according to (46), the characteristic element of maximal dimension is individuated by: $\bar{\omega}_{i}=0(i=1, \cdots, 2 \nu)$.

${ }^{82}$ See [9, p. 247] and [20, p. 429].
} 
them systematically in a new and powerful geometrical language whose central core was represented by his exterior differential calculus. At the same time, the very emphasis given by him on the language of exterior differential forms may be indicated as the main cause for the undeserved scarcity of attention that characterized for some years the response of the mathematical community towards his achievements in this field. In this connection it is interesting that still in 1924 Vessiot in [32], while praising the beauty of Cartan's integration theory, felt the necessity to translate it into its dual counterpart by replacing exterior Pfaffian forms with the notion of faisceau of infinitesimal transformations.

As Kähler suggested in the introduction to his masterpiece [21] in a really effective and historically accurate way, such a double historical origin was reflected in the twofold virtue of the theory: on one hand, with its emphasis on exterior forms, it yielded to Cartan the necessary tools for the subsequent applications to geometry (namely, the method of moving frames) as well as to the theory of infinite continuous Lie groups. On the other hand, it offered a deeper insight into the machinery (Mechanik) of partial differential equations.

\section{References}

[1] M. A. Akivis, B. A. Rosenfeld, Élie Cartan (1869-1951), American Mathematical Society, 1993.

[2] U. Amaldi, Introduzione alla teoria dei gruppi continui infiniti di trasformazioni, Parte Prima, Corsi del Reale Istituto di Alta Matematica, Roma, 1942.

[3] O. Biermann, Über $n$ simultane Differentialgleichungen der Form $\sum_{\mu=1}^{n+m} X_{\mu} d x_{\mu}$. Zeitschrift für Mathematik und Physik, 30: 234-244, 1885.

[4] A. V. Bäcklund, Über Flachentransformationen, Math. Ann., 9: 297$320,1876$.

[5] É. Cartan, Sur la structure des groupes finis et continus, Thèse, Paris, 1894.

[6] É. Cartan, Sur la réduction à sa forme canonique de la structure d'un groupe de transformations fini et continu, Amer. Jl. Math., 18: 1-61, 1896. 
[7] É. Cartan, Sur certaines expressions différentielles et le problème de Pfaff, Ann. Sci. Éc. Norm. Sup. Paris, 16: 239-332, 1899.

[8] É. Cartan, L'intégration des systèmes d'équations aux différentielles totales, Ann. Sci. Éc. Norm. Sup. Paris, 18: 241-311, 1901.

[9] É. Cartan, Sur l'intégration de certaines systèmes de Pfaff de caractère deux, Bull. de la Soc. Math. de France, 29: 233-302, 1901.

[10] É. Cartan, Sur la structure des groupes infinis de transformation, Ann. Sci. Éc. Norm. Sup. Paris, 21: 153-206, 1904.

[11] É. Cartan, Notice sur les travaux scientifiques, Paris, 1931.

[12] A. Clebsch, Über die simultane Integration linearer partieller Differentialgleichungen, J. für die reine u. angew. Math., 65: 257-268, 1866.

[13] G. Darboux, Sur le problème de Pfaff, Bulletin des sciences mathématiques, (2) 6, 14-68, 1882.

[14] É. Delassus, Extension du théorème de Cauchy aux systèmes les plus généraux d'équations aux dérivées partielles, Ann. Sci. Éc. Norm. Sup. Paris, 13: 421-467, 1896.

[15] F. Engel, Zur Invarianthentheorie der Systeme von Pfaff'schen Gleichungen, Berichte über d. Verh. d. Sächsischen Gesell. der Wiss., math.-phys. Klasse, I, 41, 157-176, 1889; II, 42, 192-207, 1890.

[16] F. Engel, Das Pfaffsche Problem, Berichte über d. Verh. d. Sächsischen Gesell. der Wiss., math.-phys. Klasse, 48, 413-430, 1896.

[17] A. R. Forsyth, Theory of differential equations. Part I. Exact equations and Pfaff's problem, Cambridge University Press, 1890.

[18] G. Frobenius, Über das Pfaffsche Problem. Jl. für die reine u. angew. Math., 82: 230-315, 1877. Reprinted in Abhandlungen 1, 249-334.

[19] E. Goursat, Leçons sur le problème de Pfaff, Librairie scientifique J. Hermann, Paris, 1922.

[20] T. Hawkins, Frobenius, Cartan, and the Problem of Pfaff. Arch. Hist. Exact Sci., 59: 381-436, 2005.

[21] E. Kähler, Einführung in die Theorie der Systeme von Differentialgleichungen, Teubner, Leipzig, 1934. 
[22] V. Katz, Differential forms - Cartan to De Rham, Arch. Hist. Exact Sci., 33: 321-336, 1985.

[23] T. A. Ivey and J. M. Landsberg, Cartan for beginners: differential geometry via moving frames and exterior differential systems, AMS Graduate Studies in Mathematics, vol. 61, 2003.

[24] S. Lie, Theorie der Transformationsgruppen. Erster Abschnitt. Unter Mitwirkung von ... Friedrich Engel, Leipzig, 1888.

[25] A. Mayer, Über unbeschränkt integrable Systeme von linearen totalen Differentialgleichungen. Math. Ann., 5: 448-470, 1872.

[26] P. J. Olver, Equivalence, Invariants and Symmetry, Cambridge University Press, 1995.

[27] P. J. Olver, Application of Lie Groups to Differential Equations, GTM Springer, Second Ed., 2000.

[28] J. M. Page, On the primitive groups of transformations in space of four dimensions, Amer. Jl. Math., 10: 293-346, 1888.

[29] C. Riquier, De l'existence des intégrales dans un système différrentiel quelconque, Ann. Sci, Éc. Norm., tome 10, 65-86 (first part), 123-150 (second part), 167-181 (third part), 1893.

[30] C. Riquier, Les systèmes d'équations aux dérivées partielles, GauthierVillars, Paris, 1910.

[31] O. Stormark, Lie's Structural Approach to PDE Systems, Encyclopedia of Mathematics, Cambridge University Press, 2000.

[32] E. Vessiot, Sur une théorie nouvelle des problèmes généraux d'intégration, Bull. Soc. Mat. Fr., 52: 336-395, 1924.

[33] E. von Weber, Zur Invarianthentheorie der Systeme der Pfaff'scher Gleichungen, Berichte über d. Verh. d. Sächsischen Gesell. der Wiss., math.-phys. Klasse, 50: 207-229, 1898.

[34] E. von Weber, Ueber die Reducirbarkeit eines Pfaff'schen Systems auf eine gegebene Zahl von Termen, Sitzungsberichte der kgl. bayer. Akademie der Wiss., 30: 273-300, 1900 (July).

[35] E. von Weber, Liniengeometrie und Pfaff'sche Systeme, Berichte über d. Verh. d. Sächsischen Gesell. der Wiss., math.-phys. Klasse, 52: 179-213, 1900 (December). 
[36] E. von Weber, Vorlesungen über das Pfaffsche Problem und die Theorie der partiellen Differentialgleichungen erster Ordnung. Teubner, Leipzig, 1900 .

[37] E. von Weber, Partielle Differentialgleichungen, Encyklopädie der Mathematischen Wissenschaften, Zweiter Band: Analysis. Leipzig, 1900.

[38] E. von Weber, Theorie der Systeme der Pfaff'scher Gleichungen, Math. Ann., 55: 386-440, 1901. 